\title{
Noise Characteristics of a Four-Jet Impingement Device Inside a Broadband Engine Noise Simulator
}

\author{
Christoph Brehm*1, \\ ${ }^{1}$ Science and Technology Corporation, Moffett Field, CA 94035 \\ Jeffrey A. Housman*2, and Cetin C. Kiris ${ }^{\dagger 2}$ \\ ${ }^{2}$ NASA Ames Research Center, Moffett Field, CA 94035 \\ Florence V. Hutcheson ${ }^{\ddagger 3}$ \\ ${ }^{3}$ NASA Langley Research Center, Hampton, VA 23681
}

\begin{abstract}
The noise generation mechanisms for four directly impinging supersonic jets are investigated employing implicit large eddy simulations with a higher-order accurate weighted essentially non-oscillatory shock-capturing scheme. Impinging jet devices are often used as an experimental apparatus to emulate a broadband noise source. Although such devices have been used in many experiments, a detailed investigation of the noise generation mechanisms has not been conducted before. Thus, the underlying physical mechanisms that are responsible for the generation of sound waves are not well understood. The flow field is highly complex and contains a wide range of temporal and spatial scales relevant for noise generation. Proper orthogonal decomposition of the flow field is utilized to characterize the unsteady nature of the flow field involving unsteady shock oscillations, large coherent turbulent flow structures, and the sporadic appearance of vortex tubes in the center of the impingement region. The causality method based on Lighthill's acoustic analogy is applied to link fluctuations of flow quantities inside the source region to the acoustic pressure in the far field. It will be demonstrated that the entropy fluctuation term in the Lighthill's stress tensor plays a vital role in the noise generation process. Consequently, the understanding of the noise generation mechanisms is employed to develop a reduced-order linear acoustic model of the four-jet impingement device. Finally, three linear acoustic FJID models are used as broadband noise sources inside an engine nacelle and the acoustic scattering results are validated against far-field acoustic experimental data.
\end{abstract}

\section{Introduction}

The current research study is part of NASA's environmentally responsible aviation (ERA) project and focuses on engine noise shielding for the reduction of community noise. The hybrid wing body (HWB) concept is currently being studied as a promising alternative to the conventional tube-and-wing aircraft due to its large payload capacity while offering enhanced aerodynamic efficiency. A key advantage of the HWB from a community noise perspective is that its fuselage can be used effectively to shield engine noise. ${ }^{1}$ Within the ERA project, a series of aerodynamic and acoustic tests of a HWB design were performed at NASA Langley Research Center. The current work is concerned with computationally reproducing the broadband engine noise simulator (BENS) experiment conducted by Hutcheson et al. ${ }^{2}$ The main purpose of this experimental investigation was to study the effects of engine placement and vertical tail configuration on shielding of broadband engine exhaust noise radiation. Figure 1 shows a CAD model of an isolated BENS which contains three so-called four-jet impingement devices (FJIDs) placed inside an engine nacelle. FJIDs are often used in aeroacoustic experiments to generate broadband noise. ${ }^{2,3}$ The three FJIDs are placed inside the nacelle in off-set positions so as to avoid generation of highly directive noise, and instead

*Research Scientist, Applied Modeling and Simulation Branch, NAS Division, MS N258-2 and AIAA Senior Member

$\dagger$ Branch Chief, Applied Modeling and Simulation Branch, NAS Division, MS N258-2 and AIAA Senior Member

${ }^{\ddagger}$ Senior Research Engineer, Aeroacoustic Branch, and AIAA Senior Member 
to promote randomization of the noise inside the nacelle before it radiates out. An extensive computational aeroacoustic analysis (CAA) of engine noise shielding is intended to follow the work presented in this paper. The shielding study will involve a large parameter space covering different airplane configurations and flight conditions requiring a large number of evaluations. Therefore, an efficient CAA approach will be required. The current study aims to obtain an efficient and accurate CAA approach that can be used for this purpose. An important part of developing this CAA approach is to obtain a reduced-order linear acoustic model of the FJID. An improved understanding of the noise generation process guides the development of this reduced order model. For this reason, a large part of this paper is concerned with studying the noise generation mechanisms of the FJID. This research study is also a continued effort towards gaining a more detailed understanding of jet impingement noise as previously studied in Brehm et al. ${ }^{4}$ for jet impingement on an inclined plate. Supersonic jet impingement problems are of great practical relevance in aeronautics and astronautics. Examples of these types of flows that have been widely studied in the literature include underexpanded jet plumes for military and commercial aircraft, jet impingement during rocket launch, initial stage of launch abort, multi-stage rocket separation, jet-engine exhaust impingement, powered Vertical Take Off and Landing (VTOL) aircraft, etc. Although jet impingement noise is of great relevance for many applications, the noise generation processes for unsteady impinging jet flows are not well understood.

To obtain an understanding of the complex fluid dynamics in the source region, proper orthogonal decomposition is employed. In Brehm et al., ${ }^{5}$ POD was previously used to identify the most energetic flow structures in a Mach 1.8 jet impinging on an inclined plate. It will be shown that the choice of the energy norm affects the coherent flow features that can be identified. While the shock motion can be effectively characterized by employing a pressure based energy norm, turbulent kinetic energy can be used to study the dynamics of shear-layers. The actual noise generation process is studied in more detail following the Lighthill's acoustic analogy. It must be noted that the physical mechanisms for acoustic sound generation in subsonic and supersonic jets has been analyzed in great detail by a large number of researchers. ${ }^{6-10}$ Following in their steps, we will utilize the causality method ${ }^{11-13}$ in the context of Lighthill's acoustic analogy to

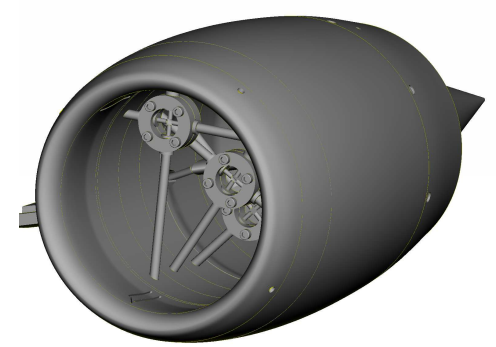

Figure 1. Three FJIDs placed inside engine nacelle (front open). identify possible noise sources in the flow field. The causality method provides a way of linking the fluctuations in the unsteady flow field to acoustic pressure fluctuations in the far-field. Different components of the Lighthill's stress tensor are cross-correlated with the acoustic pressure in the far-field.

Once adequate insight about the acoustic noise generation process is gained, a linear acoustic model is constructed to mimic the noise generated by the FJID and this model is compared to available acoustic data obtained from large eddy simulations (LES) of the FJID. For the linear acoustic analysis, an equivalent source method (ESM) described in Kiris et al. ${ }^{14}$ is chosen to solve the 3D Helmholtz equation in the frequency domain. This method is computationally very efficient and it can be employed to study acoustic scattering effects, such as engine noise shielding, ${ }^{1,2}$ for a fraction of the cost required for full CFD simulations. The linear acoustic scattering code requires either unsteady flow data on a permeable acoustic surface encompassing the source region, or an equivalent acoustic model that can effectively generate the incident pressure field in the source region. Here, we follow the latter route by creating a linear acoustic model that is able to capture the main features of the far-field acoustic pressure field of the FJID.

The current paper proceeds as follows: section II provides an overview of the numerical solver and the computational setup used to perform LES of the FJID. An evaluation of the mean flow and its unsteady flow features is provided in section III. In section V, the POD method is used to identify coherent flow features in the flow field. The causality method is introduced in section VI and utilized to obtain a link between the acoustic pressure field and the unsteady flow field in the source region. Section VII presents a linear acoustic model of the FJID employing the equivalent source method. Finally, in section VIII three linear acoustic FJID models are placed inside an isolated front-capped engine nacelle and the acoustic data is compared to experimental results.

\section{Simulation Setup}

The computational fluid dynamics (CFD) solver employed for the numerical simulations is called Launch Ascent and Vehicle Aerodynamics (LAVA). LAVA is being developed by the authors (at NASA Ames Re- 
search Center $)^{14}$ with the emphasis on solving unsteady multi-physics problems. The numerical algorithms are designed to achieve fast turn-around times for unsteady flow simulations. Optimally laying out the data structures based on the underlying adaptive mesh being used is a key aspect for achieving fast turn-around times. The LAVA solver is highly flexible with respect to the computational mesh. It supports blockstructured Cartesian meshes with Adaptive Mesh Refinement (AMR) and immersed-boundary capabilities, structured curvilinear overset meshes, and unstructured arbitrary polyhedral meshes. In order to study physically challenging flow fields such as the four-jet impingement problem, which include a wide spectrum of relevant physical time and length scales, a large number of grid points and a long time integration window with relatively small time-steps are needed. The Cartesian grid solver automatically generates volume grids given a surface triangulation or constructive solid based model. It has a fast accessible memory layout similar to that of the curvilinear solver, but cannot account for viscous and thermal effect on the walls for arbitrary geometries.

\section{II.A. Numerical Methods}

In this paper, the block-structured, Immersed Boundary (IB) AMR approach is used to simulate the jet fourjet impingement problem. This methodology is capable of automatically generating, refining, and coarsening nested Cartesian volumes. LAVA's AMR-IB method is designed to automatically generate the volume grids from a closed surface triangulation, and dynamically track important flow features as they develop. AMR is a proven methodology for multi-scale problems with an extensive existing mathematical and software knowledge base. ${ }^{15-19}$ The code has been extended using data structures and inter-level operators from the high-performance Chombo AMR library ${ }^{20}$ to provide a multi-resolution capability that can coarsen and refine the grid locally as a simulation progresses. A sharp immersed-boundary representation ${ }^{4,21,22}$ and automatic grid generation requiring only a surface triangulation make it possible to easily model complex geometries. The Cartesian mesh simulations presented herein are conducted by applying slip wall boundary conditions at all walls due to the high cost of resolving boundary layers with isotropic Cartesian cells. For the current FJID simulations, it expected that the boundary layer on the surface of the FJID does play an important role.

For the current implicit large eddy simulation (ILES), the unsteady compressible Navier-Stokes equations are solved in conservative finite difference formulation on block-structured Cartesian grids. In the Cartesian AMR solver a modified sixth-order accurate shock-capturing WENO scheme is utilized. The accuracy and computational efficiency of the higher-order shock capturing schemes within LAVA has been previously analyzed. ${ }^{23}$ A Rusanov-type flux vector splitting scheme is used on the Cartesian mesh. The viscous terms are discretized with second-order accuracy. The fourth-order accurate explicit Runge-Kutta scheme is used for time-integration. For more details about the LAVA FD solver the reader is referred to Kiris et al. ${ }^{14}$

\section{II.B. Grid Topology and Boundary Conditions}

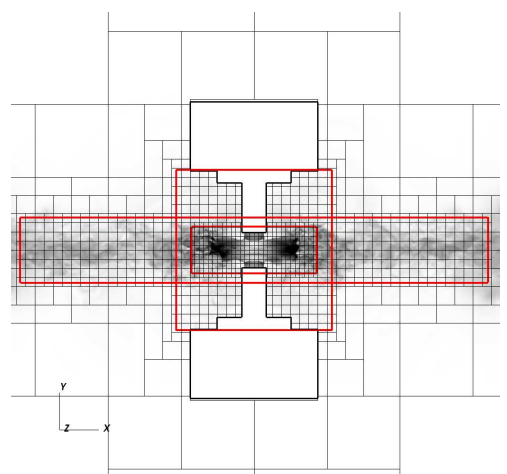

(a)

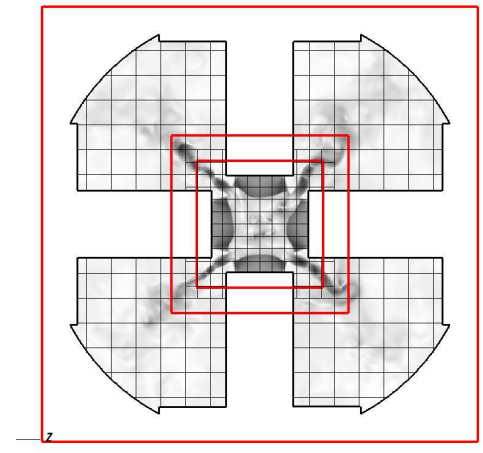

(b)

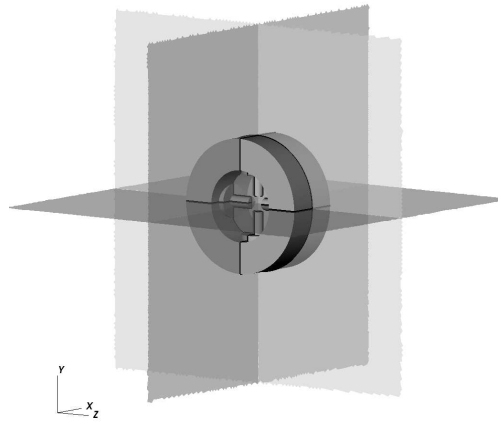

(c)

Figure 2. Computational mesh layouts in the (a) $(x, y)$-plane, and (b) $(y, z)$-plane. Refinement boxes to control the volume grid spacings are shown with red lines. (c) Two sampling planes used to gather unsteady flow data at each time-step. Grey-scale contours of Mach number in the background visualize unsteady flow field. 
Part of the computational mesh employed for the current simulations is shown in Figures $2 \mathrm{a}$ and $2 \mathrm{~b}$. Three grid resolutions are employed with 100, 180, and 300 million grid points and grid spacings of $D / \Delta x_{\min }=28.7,38.2$, and 57.3 , where $D$ is the nozzle exit diameter. Each Cartesian block (marked with solid black lines) contains $16^{3}$ grid points. Grid refinement is applied in regions where high unsteadiness and large gradients are expected, such as in the impingement region of the four jets and in the shear-layers of the deflected jets. The outlines of the volume refinement boxes to control the grid spacing in sensitive flow regions are shown in Figure 2a and $2 \mathrm{~b}$ as red solid lines. Since storing the entire volume data for each time-step requires excessively large memory, three sampling planes (displayed in Figures 2c) were used to gather the unsteady flow data at each time-step. Most of the unsteady flow analysis was applied to the data recorded in these sampling planes. The total mass-flow rate at the exits of the four nozzles exits is $0.15 \mathrm{lb} / \mathrm{s}$. The mass-flow rate was chosen based on values reported from the experimental set-

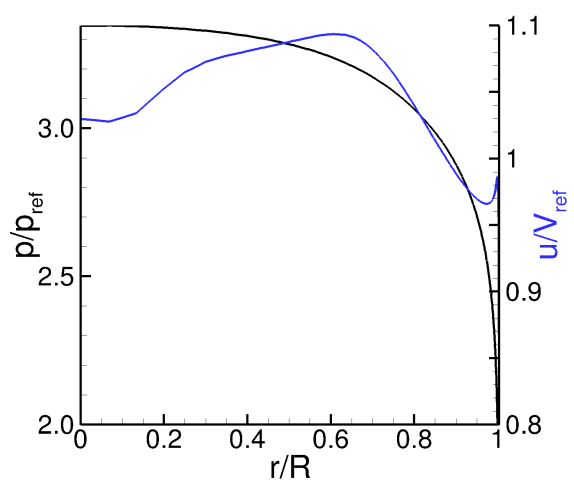

Figure 3. Streamwise velocity (in $\mathrm{m} / \mathrm{s}$ ) and pressure (in Pascals) profiles at the nozzle exits. ting. ${ }^{24}$ The streamwise velocity and pressure profiles at the exits of the four nozzles were obtained in an independent simulation with our in-house unstructured solver within LAVA. ${ }^{14}$ The inflow profiles for the unsteady simulations are provided in Figure 3. Note that throughout this paper all flow quantities were normalized with mean flow quantities at the nozzle exit, i.e., exit velocicty, $V_{\text {ref }}$, pressure, $p_{\text {ref }}$, and temperature, $T_{r e f}$ (averaged over the nozzle exit). The Reynolds number based on $D, V_{r e f}$ and $T_{r e f}$ is approximately 75,000 .

\section{II.C. Computational Simulation Strategy}

The current computational simulation strategy is closely aligned with the findings by Shur et al. ${ }^{25,26}$ with respect to nozzle inflow conditions and more importantly subgrid-scale modeling for jet flow-acoustics simulations. With simulation approach Shur et al. ${ }^{25,26}$ demonstrated excellent agreement between their jet noise predictions and experimental data. It is well known that direct numerical simulations (DNS) are limited to Reynolds numbers of up-to $\mathcal{O}\left(10^{4}\right)$. Hence, since DNS is out of reach for the current flow conditions, a large eddy simulation (LES) approach is utilized as it has been common practice for similar flow conditions, where molecular viscosity has little effect and likely less than the inflow shear layer thickness or state (laminar versus turbulent) as suggested by Shur et al. ${ }^{25}$

In the larger context of LES, for the current simulations, an implicit large eddy simulation (ILES) is used. In contrast to classical LES, which employ an explicitly computed sub-grid scale (SGS) closure, ILES relies on the inherent regularization mechanism through the truncation error of the convective fluxes also referred to as implicit SGS model. ${ }^{27}$ The numerical scheme must be chosen carefully to provide a physically consistent implicit SGS model. In the current study, the modified sixth-order accurate WENO scheme ${ }^{28}$ was used due to its superior physically motivated scale separation properties. Hu and Adams ${ }^{27,28}$ have demonstrated that the modified sixth-order accurate WENO scheme reproduces the Kolmogorov range of the turbulent kineticenergy spectrum at the limit of infinite Reynolds number, independent of grid resolution while retaining the shock-capturing capabilities of the original WENO-CU6. Figure 4a and 4b presents instantaneous color contours of gauge pressure and solid contour lines of density gradient utilizing the higher-order WENO scheme and a second-order accurate MUSCL scheme. When comparing the flow features between the two solutions, it can be noted that the second-order solution is significantly more dissipative than the higher-order solution. A detailed discussion on the higher-order shock capturing scheme utilized here and its numerical properties is provided in Brehm et al..$^{23}$ It is important to point out that the authors' experience, as well as other research studies, ${ }^{5,25,26}$ confirm that for simulations of turbulent flows away from physical boundaries the ILES approach with its subtle numerical dissipation follows the basic physical principles of LES (see also $\mathrm{Hu}$ et $\left.a{ }^{27}{ }^{27}\right)$. Moreover, the approach is efficient in utilizing the available grid resolution for capturing the shear-layer transition process and modeling salient features of the turbulent flow. Preliminary studies by other researchers ${ }^{25,29}$ demonstrated that the transition of the shear-layer may be delayed and that the turbulence was not captured as well when utilizing an explicit SGS models. In Brehm et al.??, the authors demonstrated that with the present ILES approach an excellent agreement between with far-field acoustic measurements by ${ }^{30}$ was obtained. 


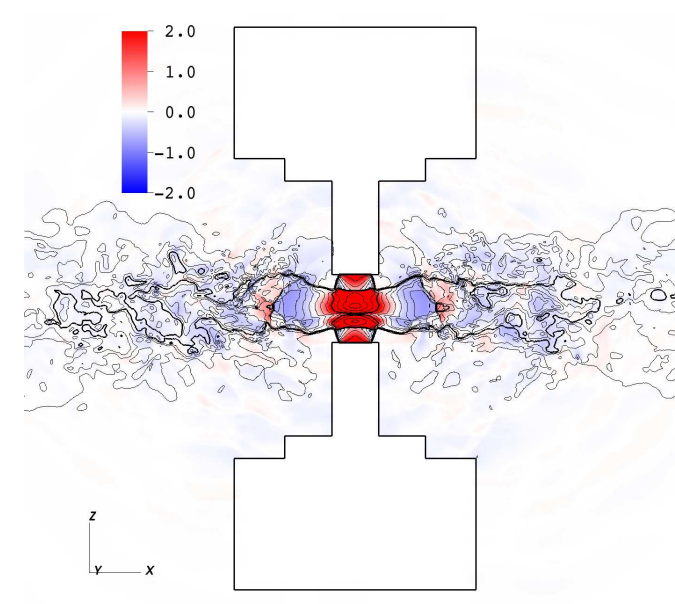

(a) WENO

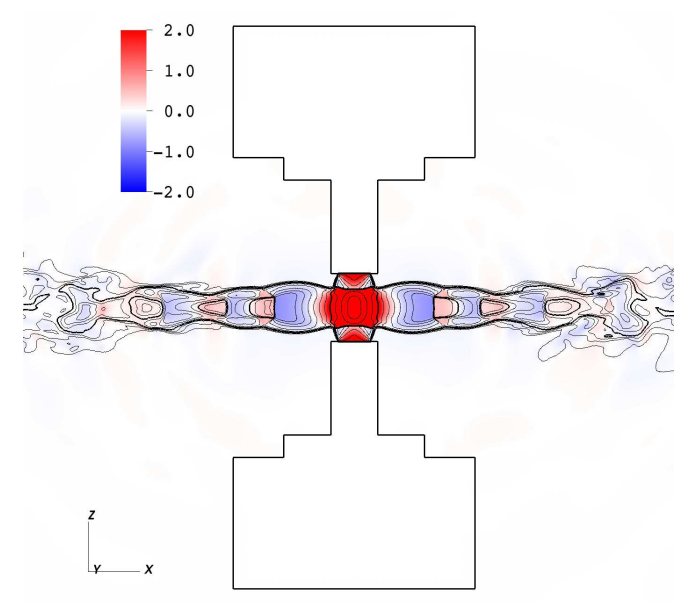

(b) MUSCL

Figure 4. Instantaneous color contours of gauge pressure and solid contour lines of density gradient for numerical solutions with (a) WENO and (b) MUSCL.

Another important detail of the computational setup is the nozzle inflow boundary conditions specification. Ideally, the nozzle exit flow contains a fully resolved turbulent boundary layer convecting unsteadiness into the jet's shear layer. Since the needed computational resources are not available for such a simulation, we are relying on the fact that the shear layer will essentially transition and create a more energetic unsteadiness (ultimately turbulent flow state) in the jet than in the nozzle boundary layer flow. The approximate thickness of the boundary layer was captured by employing a RANS model inside the pipe and the actual nozzle nozzle. The transition process of the shear layer is only mildly affected by the (fine scale) turbulence in the nozzle boundary layer while the displacement thickness of the boundary layer has a stronger influence. ${ }^{25}$ Note that some researchers ${ }^{9}$ apply some form of unsteady forcing to seed unsteadiness in the transitional shear layer. One natural choice would be to introduce perturbations based on linear stability theory, ${ }^{31}$ i.e., Tollmien Schlichting waves. It is, however, cumbersome to achieve a realistic scenario with respect to instability wave frequencies and amplitudes, which would also require a significant spatial extend for the initial disturbances to develop. Hence in the present study any type of forcing based on linear stability theory and/or random noise was avoided to eliminate the dependence of the simulation on free parameters. Studies regarding the effect of this inflow forcing on jet noise can be found in Lew et al. ${ }^{32}$ and Bogey and Bailly. ${ }^{33}$ The elevated acoustic background noise arising in jet impingement continuously seeds the unsteadiness in the shear layer. Moreover, comparing the current grid resolution with computational setups of similar research studies suggests that the current grid resolution is sufficient to study the underlying flow physics. Sufficient grid convergence of the relevant flow physics will also be demonstrated by a grid resolution study provided in section IV.A.

\section{Main Flow Features}

Color contours of gauge pressure and solid line contours of Mach number with a thicker solid line marking the sonic line are shown in figures $5 \mathrm{a}$ and $5 \mathrm{~b}$. The time-averaged flow field provides an overview of some of the key flow features for this unique flow scenario. As can be seen in figure 5a, two primary deflected supersonic jets form along the x-direction carrying most of the momentum flux introduced by the four nozzles. In addition to these two primary deflected jets, the four directly impinging jets in figure 5b create four secondary jets diagonally ejecting into the gaps between the nozzles of the FJID. Based on the large disparity in the amount of momentum flux between the two primary jets in $\mathrm{x}$-direction and the four diagonal jets in the $(y, z)$-plane, it is expected that the two jets in $\mathrm{x}$-direction are the main contributors to the acoustic noise field. The flow field contains complicated shock structures that are highly unsteady as the instantaneous

flow visualization in figure 4a illustrates. The two primary jets break up quickly due to strong unsteadiness and essentially only two shock cells remain in the time-averaged flow field of the jets. 


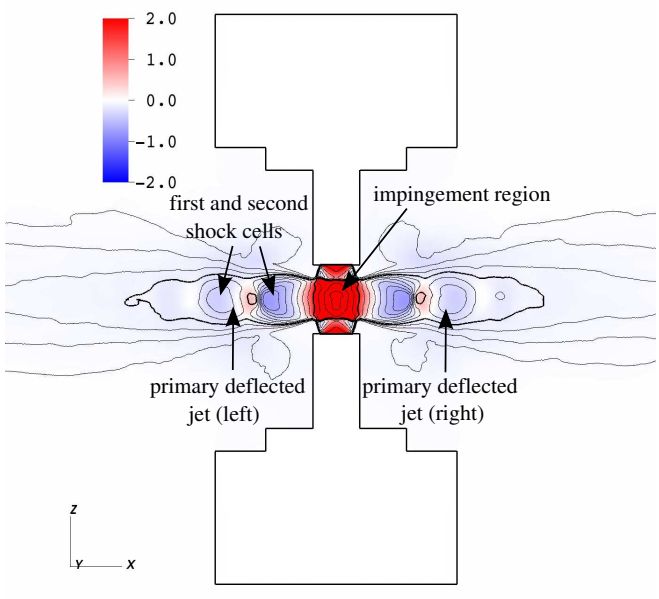

(a)

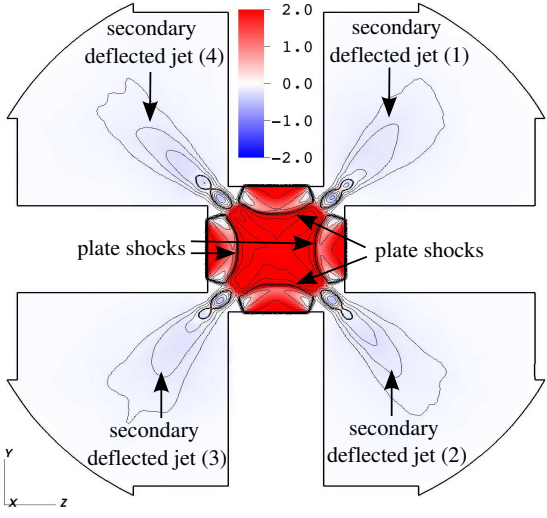

(b)

Figure 5. Overlay of colored contours of pressure, contour lines of Mach-number and the sonic line marked as thick, black solid line within the time-averaged four-jet impingement device.

\section{Unsteady Flow Features}

\section{IV.A. Grid Resolution Study}

The sensitivity of the CFD simulation results with respect to the grid resolution is investigated by comparing the power-spectra of pressure $E_{p}$ at five sample points inside the source region as marked in figure $6 \mathrm{a}$. To allow for a better comparison of the power-spectra for the different grid resolutions, the spectra were smoothed using simple averaging. A significant increase in the spectral energy can be noted from the coarse grid to the medium grid. The difference in the power spectra $\left(\Delta E_{p}\right)$ is significantly smaller between the medium and fine grids. The grid convergence study considering the power spectra of pressure in the near field indicate sufficient grid convergence. Further, comparisons of other relevant flow quantities (not shown here) on the coarse, medium, and fine grids convey the same message.

In addition to the grid sensitivity, the different power-spectra provide some idea about the frequency characteristics in different parts of the flow field. In the shear-layer the power-spectra of pressure for sample points 1 and 5 contain broadband spectra that are relatively flat until $S t \approx 1$. The power-spectra of pressure inside the jet impingement region (sample point 4) and the end of the first shock diamond structure in the deflected jets (sample points 3) contain significantly more energy. The unsteadiness in this region is dominated by lower frequency content $(S t<0.1)$. Sample point 2 is placed in the corner of the impingement region at the inception of the shear-layer of the primary deflected jets (in $x$-direction). The power-spectra for sample point 2 contains a more pronounced peak around $S t=0.1-0.2$. All five power spectra display relatively broadband spectral characteristics in the source region.

\section{IV.B. Reynolds Stresses and Entropy Fluctuations}

To obtain a general overview of the unsteady flow features in the flow field the Reynolds stress tensor components, $R_{i j}=\left\langle u_{i}^{\prime} u_{j}^{\prime}\right\rangle$ with $u_{i}^{\prime}=u_{i}-\left\langle u_{i}\right\rangle$, and the entropy fluctuation term, $\left\langle\left|p^{\prime}-a_{\infty}^{2} \rho^{\prime}\right|\right\rangle$, based on Lighthill's acoustic analogy are computed. In the above expressions, $\left\langle u_{i}\right\rangle$ refers to the mean flow velocity component and $u_{i}^{\prime}$ is the disturbance velocity components. Color contours of the Reynolds stress tensor and entropy fluctuation term are presented in figures $7 \mathrm{a}-\mathrm{h}$. Note that the disturbance flow velocity vector was locally decomposed into three components, $u_{1}^{\prime}$ (direction of the mean flow), $u_{2}^{\prime}$ (perpendicular to the mean flow inside the sampling plane), and $u_{3}^{\prime}$ (perpendicular to the sampling plane). Thus, it consequently follows $\left\langle u_{2}\right\rangle=0$.

Visualizing the Reynolds stress tensor components and the entropy fluctuation term highlights regions with high fluctuation amplitudes. This analysis provides some initial insight on the underlying physical

mechanisms responsible for the noise generation. Since the main structure of the two primary jets breaks up quickly the region with large unsteady fluctuations is confined within a small area around the FJID. 


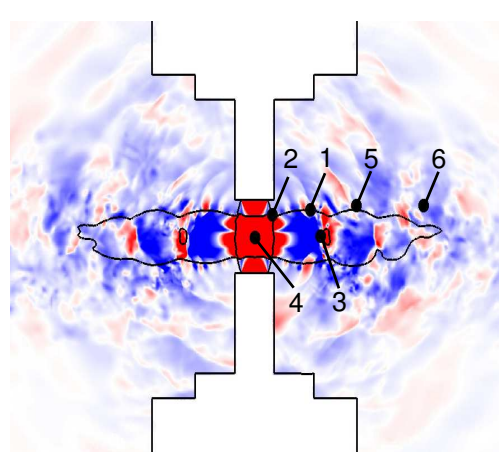

(a)

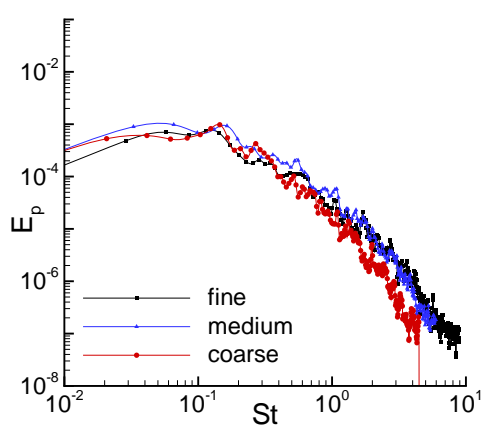

(d)

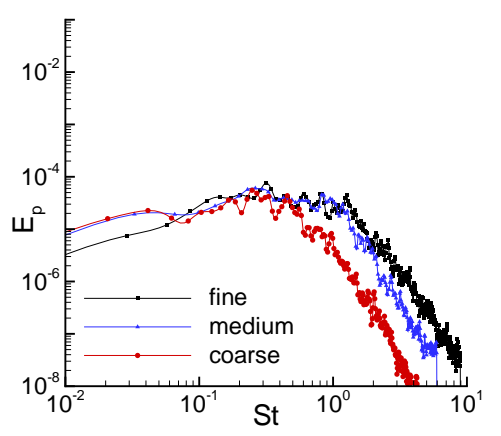

(b)

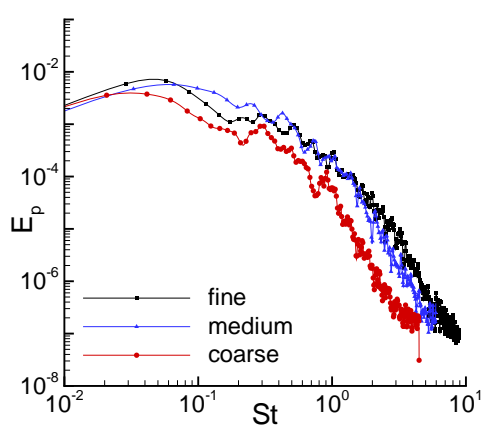

(e)

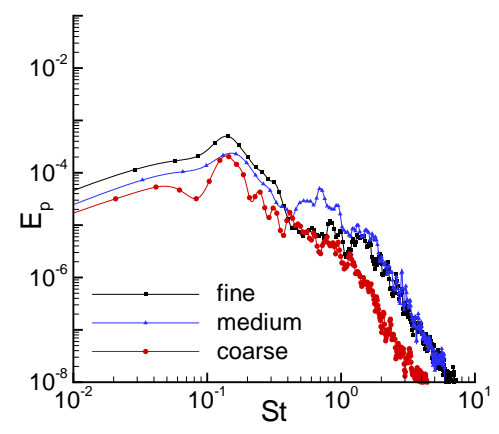

(c)

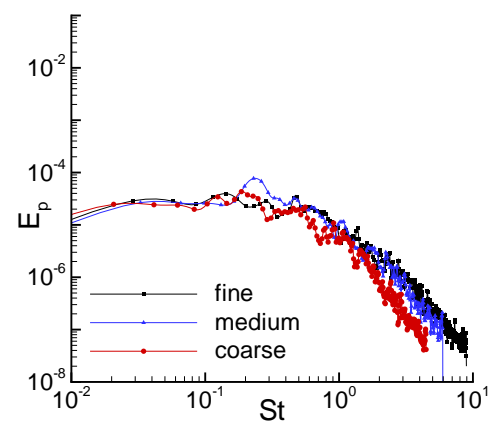

(f)

Figure 6. (a) Sample points (1-6) in the unsteady flow field. (b-f) Power spectra of unsteady pressure field at sample points (1-5).

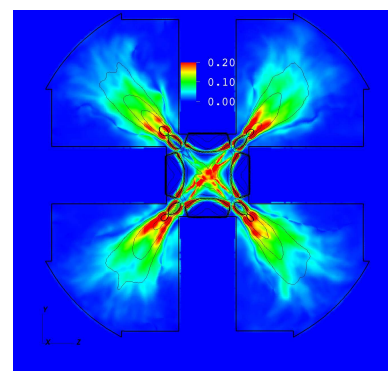

(a) $\left\langle u_{1}^{\prime} u_{1}^{\prime}\right\rangle$

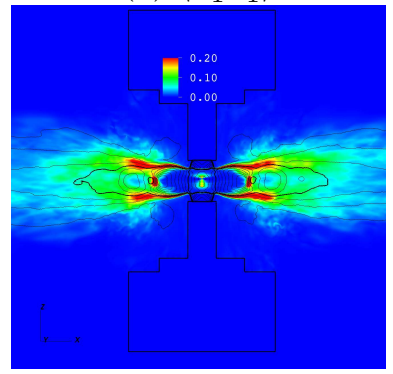

(e) $\left\langle u_{1}^{\prime} u_{1}^{\prime}\right\rangle$

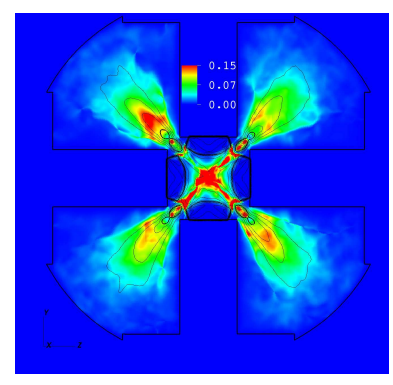

(b) $\left\langle u_{2}^{\prime} u_{2}^{\prime}\right\rangle$

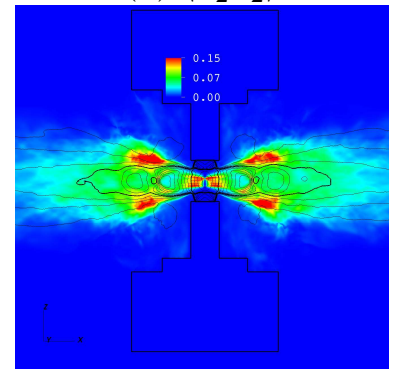

(f) $\left\langle u_{2}^{\prime} u_{2}^{\prime}\right\rangle$

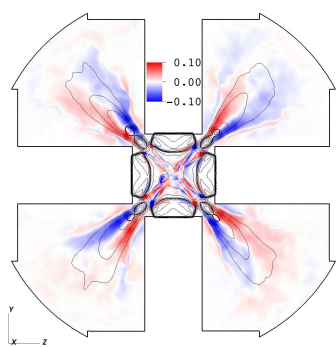

(c) $\left\langle u_{1}^{\prime} u_{2}^{\prime}\right\rangle$

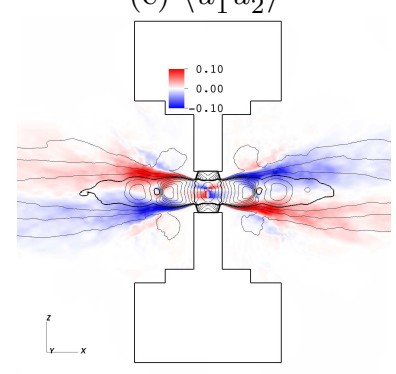

(g) $\left\langle u_{1}^{\prime} u_{2}^{\prime}\right\rangle$

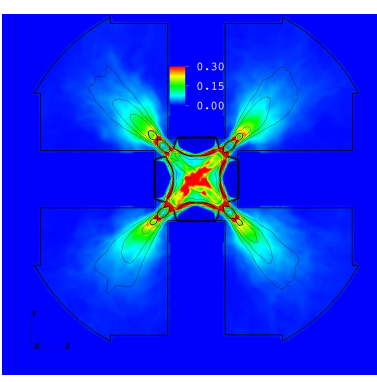

(d) $\left\langle\left|p^{\prime}-a_{\infty}^{2} \rho^{\prime}\right|\right\rangle$

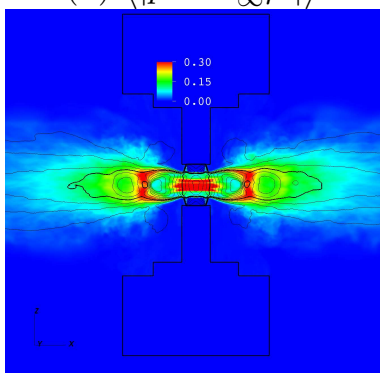

(h) $\left\langle\left|p^{\prime}-a_{\infty}^{2} \rho^{\prime}\right|\right\rangle$

Figure 7. Colored contours of different Reynolds stress tensor components, $R_{i j}=\overline{u_{i}^{\prime} u_{j}^{\prime}}$, and the entropy fluctuation term.

Large normal Reynolds stress tensor components are identified inside the jets' shear-layers and inside the impingement region. Larger normal Reynolds stresses are observed in the streamwise direction $\left\langle u_{1}^{\prime} u_{1}^{\prime}\right\rangle$ in comparison to the cross-streamwise component $\left\langle u_{2}^{\prime} u_{2}^{\prime}\right\rangle$, which is common for non-isotropic turbulence in 
shear-layers. Large amplitudes of the off-diagonal component $\left\langle u_{1}^{\prime} u_{2}^{\prime}\right\rangle$ is due to unsteady vortical flow structures inside the shear-layer. Furthermore, in the unsteady flow visualization a flapping motion of the primary and secondary jets was noted. Large amplitudes of the entropy fluctuation term are observed around the first shock diamond and inside the jet impingement region. The unsteadiness in the primary jets' shear-layers induces an unsteady motion of the Mach diamond structure causing significant fluctuations at the Mach disk of the first shock cell. The large entropy fluctuations in the center of the jet impingement region are caused by strong vortex tubes that are generated at the edge of the four-jet impingement region. These longitudinal vortices gravitate towards the center of the impingement region. Traces of these vortices can be identified in all components of the Reynolds stress tensor. To the best knowledge of the authors, the occurrence of these flow structures has not been reported previously. In order to ensure the validity of our numerical simulations we ran a number of variations of the simulation setup, employing different numerical fluxes, WENO schemes, and time-integration method. The current analysis only highlights regions with large unsteady fluctuations. It does not differentiate between sound producing and "silent" unsteadiness. Comparing the Reynolds stresses and the entropy fluctuation term in figure 7 with the non-normalized crosscorrelation analysis results discussed in section VI provides an idea on what fraction of the unsteady flow field is producing acoustically radiating pressure waves.

\section{IV.C. Intermittency}

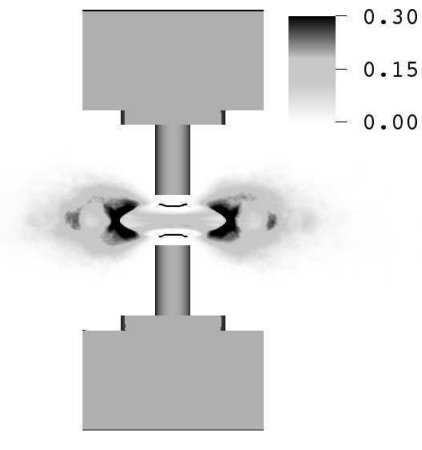

(a)

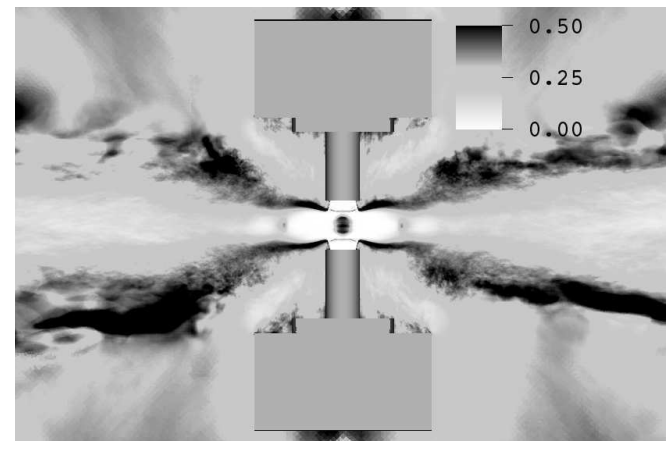

(b)

Figure 8. Grey-scale contours of the intermittency factor $\gamma$ for (a) disturbance pressure and (b) turbulent kinetic energy.

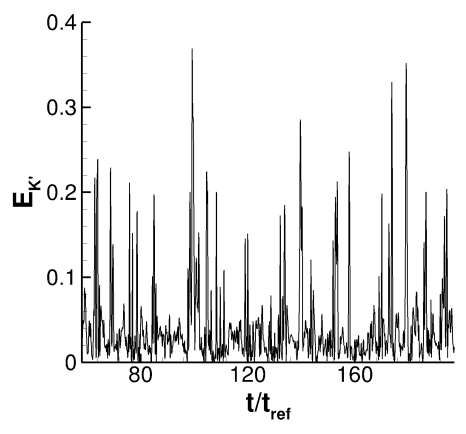

(a)

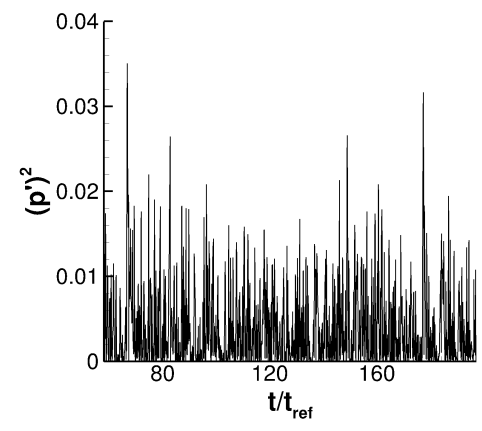

(b)

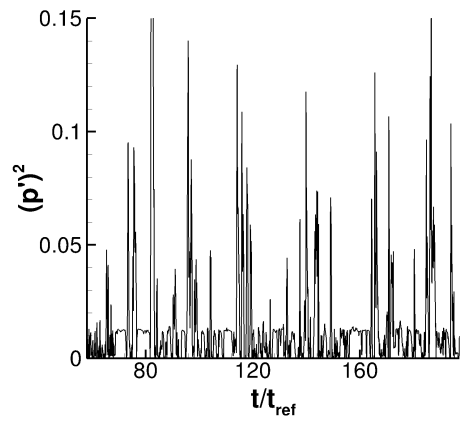

(c)

Figure 9. Time-signals of (a) $E_{K}^{\prime}$ at the edge of the impingement region (sample point 2), (b) $\left(p^{\prime}\right)^{2}$ in the shear layers of the primary deflected jets (sample point 1), and (c) $\left(p^{\prime}\right)^{2}$ in the vicinity of the Mach disk of the first shock cell.

The unsteady flow visualization clearly depicts the occurrence of intermittent events that cause large pressure fluctuations in the flow field. In the present work, the intermittency of different disturbance flow quantities is evaluated by computing the intermittency factor in the entire $(x, y)$-sample-plane. In this work, 
the intermittency factor is defined by using function $I(t)$ in the form

$$
I(t)= \begin{cases}1 & \text { if } q<0.5\langle q\rangle \\ 0 & \text { otherwise }\end{cases}
$$

The intermittency factor is then defined as $\gamma=\langle I(t)\rangle$. The intermittency maps for disturbance pressure, $q=\left(p^{\prime}\right)^{2}$, and turbulent kinetic energy, $q=0.5\left(\left(u^{\prime}\right)^{2}+\left(v^{\prime}\right)^{2}+\left(w^{\prime}\right)^{2}\right)$, are shown in Figure 8. It is assumed that $\gamma \approx 0$ when the flow is laminar or fully turbulent and $\gamma \approx 1$ when the signal is intermittent. The intermittency map for disturbance pressure shows intermittent events occurring around the Mach disk of the first shock cell in the primary jets as well as an intermittent motion of the plate shocks of the four jet impingement region. For turbulent kinetic energy, a larger region of the flow field appears to be intermittent in comparison to the disturbance pressure field. The intermittency map for turbulent kinetic energy highlights the shear layers of the primary jets and the center of the four jet impingement region. Sample time-signals of disturbance pressure and turbulent kinetic energy are shown in figures 9a-c. The time-signal of $\left(p^{\prime}\right)^{2}$ at probe 1 is provided to give an idea of a less intermittent signal for this flow field. The other two time-signals clearly display an intermittent behavior. This begs the question of whether temporal Fourier analysis is the right tool to analyze the unsteady character of the flow field. For this reason wavelet transforms are used in the next section to provide an additional perspective on the highly intermittent flow features.

\section{IV.D. Wavelet Transform}

Wavelet transforms offer a different view on analyzing the unsteady flow field. While the location information, i.e., here the location in time, is generally lost in the Fourier transform, wavelet transforms capture both frequency and location information. By employing wavelet transforms we are able to scrutinize the occurrence of intermittent (or sporadic) events that were discussed in the previous section. A discrete wavelet transform (DWT) using Ricker wavelets was applied to the time-signals recorded at the point probes shown in figure 6a. The resolution of the wavelets is mapped to a scale that can be interpreted as a pseudo-frequency, here denoted by

$$
\tilde{S t}=\frac{S t_{c}}{a T_{s}}
$$

In equation (2), the pseudo-frequency, $\tilde{S} t$, is computed from the sampling period, $T_{s}$, the center frequency, $S t_{c}$, and the scale $a$ of the wavelet. In this context, it is assumed that the wavelet can be associated with a harmonic time-signal of frequency $S t_{c}$. For the current results, the center frequency is given by the frequency that maximizes the Fourier transform of the wavelet modulus.

Figures 10a-f present colored contours of wavelet transformed time-signals of pressure recorded at probes 1-6 in figure 6a. The pseudo frequency as well as the amplitude associated with these peaks significantly varies over time and does, therefore, not display the typical signature that would be associated with harmonic time-signals. The DWT of the time-signal at point probe 1 contains a large amount of high-frequency content which is typical for high-Reynolds number shear-layers. The pressure time-signal at this sample point is less intermittent as has been noted in the previous section IV.C. The Mach disk motion of the first shock cell is dominated by lower frequencies as can be seen in the DWT of the time-signal at point probe 3 . The appearance of intermittent events can clearly be identified in the DWTs. Point probe 4 in the center of the jet impingement region captures the formation of pressure pulses that affects the time-signals at all point probes. In the current DWT analysis, the occurrence of intermittent events is further scrutinized by visualizing the flow field at positions in time when these events are present in the DWT contours in figure 10.

Figure 11 displays instantaneous contours of gauge-pressure and contour lines of dilatation at $t / t_{r e f}=80$, 105 , and 124. The flapping motion of the primary jets causes the the first shock cell to move towards sample point 5 inducing large pressure amplitude changes. Large pressure fluctuations are intermittently being generated in the center of the four-jet impingement region. The large amplitude in the DWT at $t / t_{\text {ref }}=124$ at probe 4 is associated with a vortex tube being located right above the sensor.

In the more conventional approach of analyzing unsteady flow fields, the time-signals are studied employing Fourier transforms that do not reveal this time behavior. FFT data can be compared with the DWT data by computing a DWT pseudo spectrum. Figure 12 shows a comparison of DWT with FFT data. In 


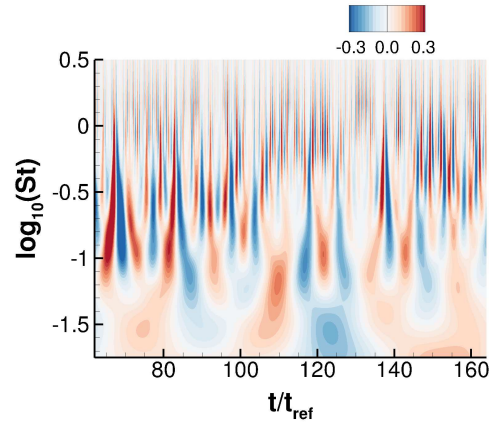

(a) probe 1

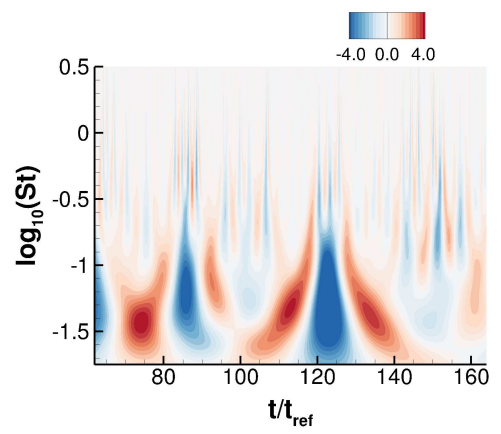

(d) probe 4

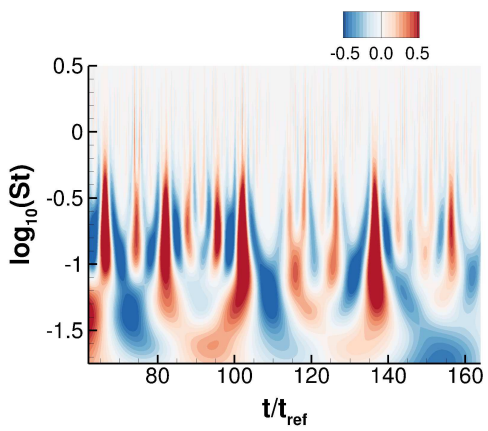

(b) probe 2

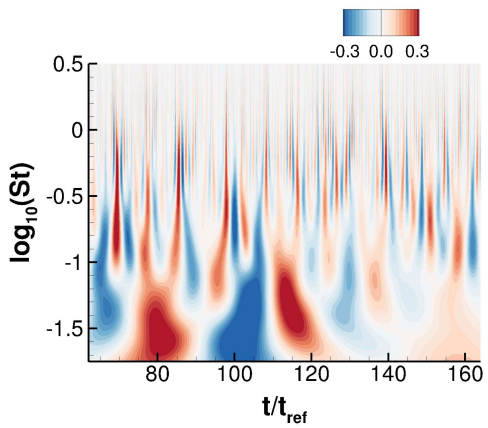

(e) probe 5

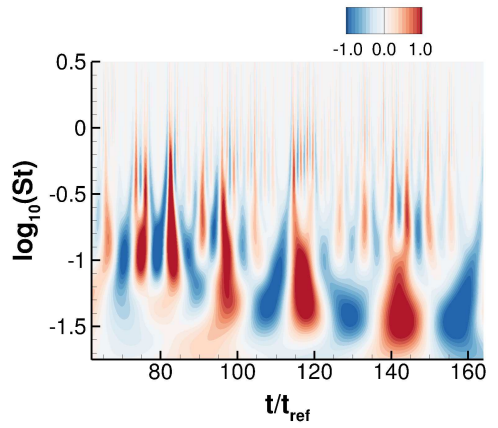

(c) probe 3

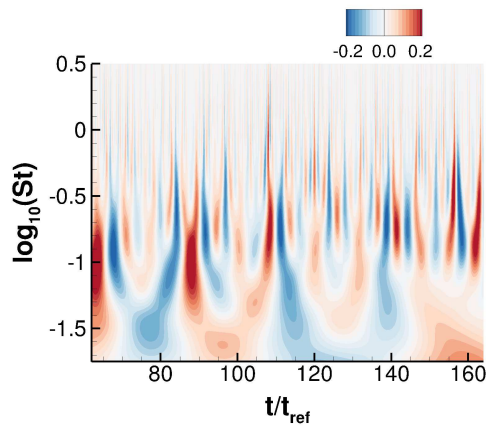

(f) probe 6

Figure 10. Colored contours of wavelet transformed time-signals of pressure recorded at probes 1-6 (a-f) versus nondimensional time and pseudo Strouhal frequency in log-scale.

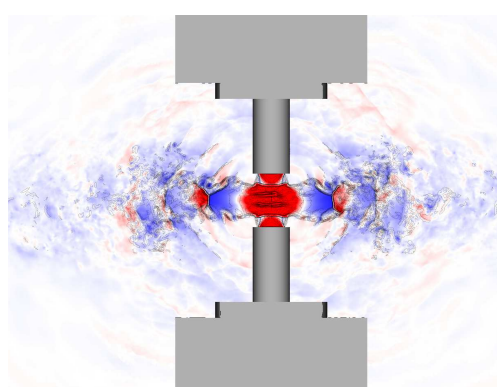

(a) $t / t_{\text {ref }}=80$

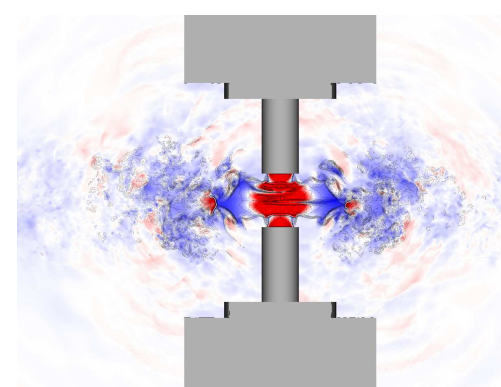

(b) $t / t_{\text {ref }}=105$

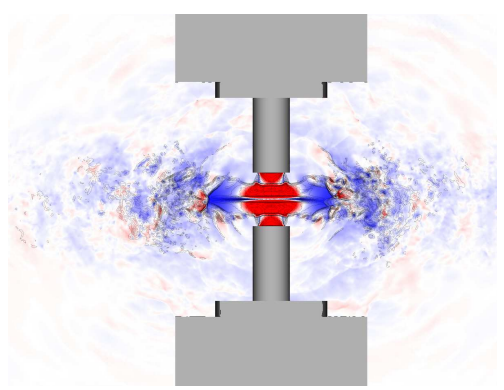

(c) $t / t_{r e f}=124$

Figure 11. Instantaneous contours of gauge-pressure and contour lines of dilatation at $t / t_{r e f}=80,105$, and 124 .

the time interval $t \in\left[t_{1}, t_{2}\right]$, the DWT pseudo spectrum was simply computed with

$$
A_{D W T}(\tilde{S} t)=\alpha \sqrt{\frac{\int_{t_{1}}^{t_{2}} A^{2}(\tilde{S} t, t) d t}{t_{2}-t_{1}}},
$$

where $\alpha$ is a constant scaling factor to obtain amplitudes that are comparable to FFT amplitudes. Overall the integral DWT amplitudes match the general trends of the FFT coeffients very well. Hence, the definition of the DWT pseudo Strouhal number appears to be consistent with the FFT results. This allows an interpretation of the frequency characteristics over time as displayed in figure 10. An advantage of the smooth integral DWT amplitude curves is that it allows for a more straightforward comparison between different data sets. For intermittent flow fields the DWT provides information about when intermittent events occur while in addition the frequency information in the sense of FFT remains. 


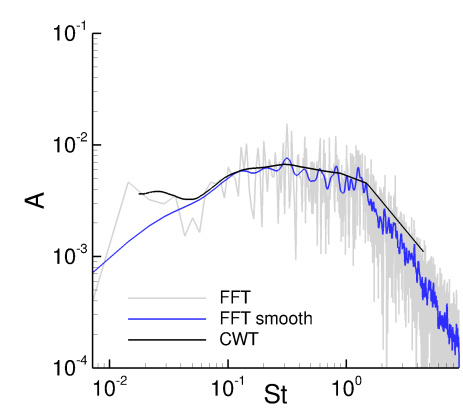

(a) probe 1

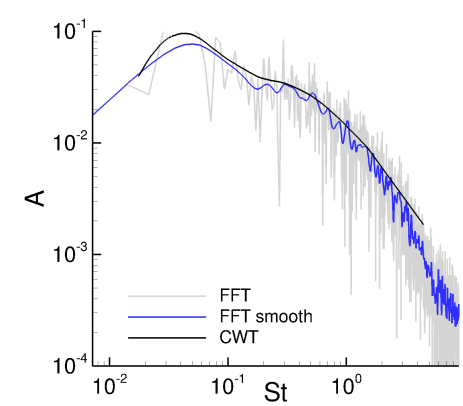

(d) probe 4

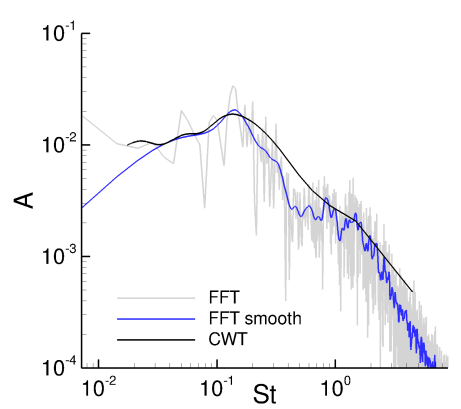

(b) probe 2

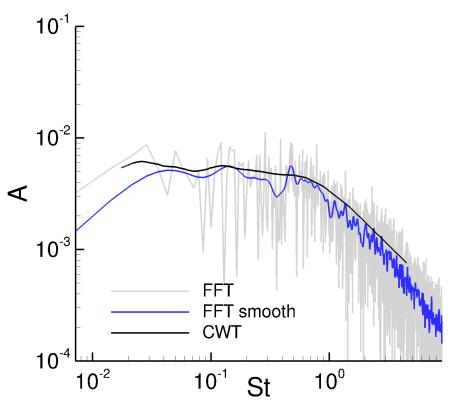

(e) probe 5

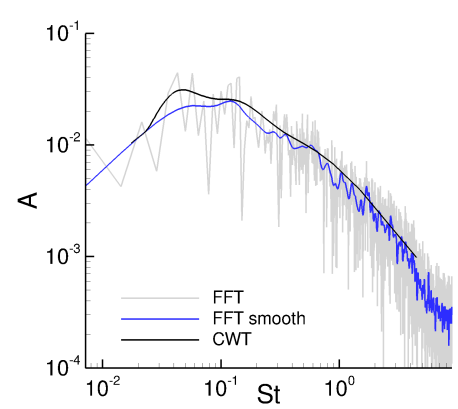

(c) probe 3

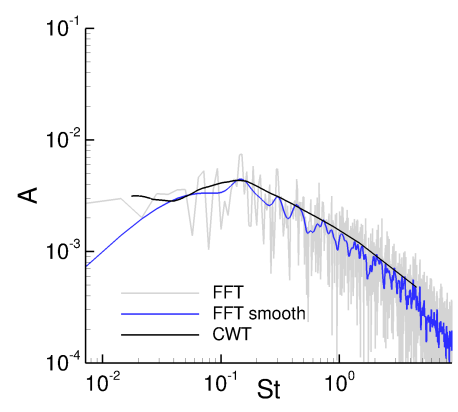

(f) probe 6

Figure 12. Integral DWT amplitude defined by equation (3) and magnitude of FFT coefficients at different point probes 1-6.

\section{Proper Orthogonal Decomposition}

POD decomposes the flow field into a set of orthogonal basis functions that capture most of the flow energy in terms of a user-defined norm with the least number of modes. ${ }^{34}$ In many fluid dynamics applications, POD is used to identify energetic flow structures. A dynamical significance of existing POD modes is not guaranteed. Freund and Colonius ${ }^{35}$ applied POD to a Mach 0.9 turbulent jet. and noted that the sound radiated by turbulence amounts to a very small fraction of the total energy. Thus, a POD approach in the temporal domain may not be able to capture the physics of the radiated sound waves. When analyzing the acoustic field it may be advantageous to follow an approach similar to the one described in Suzuki et $a l .{ }^{36}$ Instead of using snapshots in time, Suzuki et al. ${ }^{36}$ used Fourier amplitude distributions as snapshots for POD. In the current study, we are dealing with mainly broadband noise sources as well as intermittent events and, therefore, POD was applied in the temporal domain. The frequency information of dominant unsteady jet and shock dynamics is obtained by applying a Fourier and wavelet transform to the POD time-coefficients.

Furthermore, the appropriate norm for capturing particular flow physics is not necessarily clear. Freund and Colonius ${ }^{35}$ have found that the effectiveness of a given representation of the initial flow data depends strongly upon the norm used and the data represented. For problems where the spatial dimension, $m$, is much greater than the number of available time-steps, $N$, it is more efficient to employ the "snapshot" method $^{37}$ for POD. Hence, in the current analysis the snapshot method is used. For more details about the POD snapshot method used here, see Chatterlee ${ }^{38}$ for a basic introduction to POD, and Rowley ${ }^{39}$ and Freund and Colonius ${ }^{35}$ on details on how it can be applied to fluid dynamics problems. In the current discussion, it is assumed that a vector of flow quantities, here $\vec{q}(\vec{x}, t)=\left(p^{\prime}, u^{\prime}, v^{\prime}, w^{\prime}, s^{\prime}\right)$, is defined over a region of interest $\Omega$ and additional weighting factors $\vec{\omega}$ weigh the contribution of the components of $\vec{q}(\vec{x}, t)$ in the vector norm,

$$
|\vec{q}|_{\Omega}^{2}=\int_{\Omega}\left(\sum_{k=1}^{N_{q}} \omega_{k} q_{k}^{2}\right) d \vec{x},
$$

where $N_{q}$ is the number of components of $\vec{q}$. By employing POD the series expansion converges optimally 
with respect to the $\mathrm{L}_{2}$-norm defined in Equation (4). Kinetic disturbance energy, the entropy fluctuation term, pressure or other combination of $\vec{q}$ can be used in the $\mathrm{L}_{2}$-norm by choosing the weighting vector as $\vec{\omega}=(0,1,1,1,0),(0,0,0,0,1)$, and $(1,0,0,0,0)$, respectively. In Brehm et al. ${ }^{5}$ it was shown that computing the most energetic $p^{\prime}$-based POD modes is useful for obtaining a general sense of the unsteady shock motion for jet impingement problems. In order to study the noise generation process $s^{\prime}$-based and $K^{\prime}$-based POD modes provide some idea of how noise is generated in the source region when considering that the general structure of Lighthill's stress tensor is composed of $\rho u_{i}^{\prime} u_{j}^{\prime}$ and the entropy fluctuation term $s^{\prime}$. More details about the computation of the POD modes and singular values associated with each mode are provided in appendix A.

\section{V.A. POD Results}

In order to measure the coherence in the flow field the coherence factor $\psi_{N}$ is introduced. This factor provides a measure for how much energy in the pressure field is captured by the first $N$ POD modes:

$$
\psi_{N}=\left(\sum_{m=1}^{N} \lambda^{n}\right) /\left(\sum_{m=1}^{N_{t o t}} \lambda^{n}\right),
$$

where $\lambda^{n}$ are the eigenvalues of the eigenvalue problem described in equation (12), $N_{\text {tot }}$ is the total number of POD modes and $N<N_{t o t}$. For jet impingement flows, ${ }^{23}$ it is very common for a large amount of energy to be contained in a few number of POD modes due to the intensity of the pressure oscillations in the impingement region. Figure 13 shows that for the four-jet impingement problem roughly 40 POD modes are

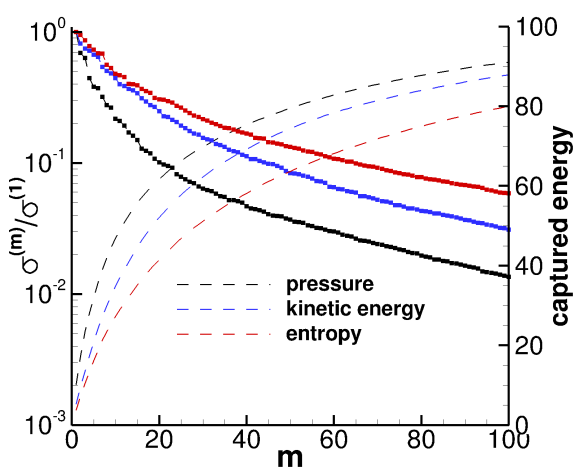

Figure 13. Singular values and total energy captured by the first N POD modes. sufficient to capture $80 \%$ of the energy defined by the $\mathrm{L}_{2}$-norm of pressure in equation (4). The pressure field appears to be significantly more coherent than when basing the energy norm on kinetic disturbance energy or the entropy fluctuation term. Figure 14 displays the three most energetic $p^{\prime}$-based, $K^{\prime}$-based, and $s^{\prime}$-based POD modes. Depending on the choice of the energy norm, different characteristics of the unsteady flow field can be identified. The pressure field is dominated by significant pressure oscillations inside the four-jet impingement region. The most energetic $K^{\prime}$-based POD modes highlight the shear-layers of the two primary jets that interact with the shock cells inside the jets. The coherence of $K^{\prime}$-based POD modes quickly breaks up in downstream direction shortly downstream of the first shock cell. A coupling between the entropy fluctuations in the center of the four jet impingement region, around the jets' shear layers, and the Mach disk of the first shock-cell can also be identified for the most energetic $s^{\prime}$-based POD modes. The pressure field is dominated by highly-energetic pressure fluctuations in the center of the four jet impingement region. $K^{\prime}$-based and $s^{\prime}$-based POD modes appear to capture a complicated coupling between the four-jet impingement region, the shear-layers of the primary jets, and the dynamics of the shock-cell structures.

Lastly, the analysis of the temporal behavior of the different POD modes remains. The time-coefficients in equation (14) are Fourier transformed in time and the Fourier spectra are shown in figures 15a-c. The Fourier and DWT pseudo spectra for the $p^{\prime}$-based POD modes are relatively broad due to the intermittent unsteady nature of the flow field in the impingement region. The Fourier and DWT pseudo spectra for the $K^{\prime}$-based and $s^{\prime}$-based POD modes display a broadband peak around $S t=0.2$. The broadband peak at $S t=0.2$ may be associated with a dominant frequency in the shear layer of the primary jets. 


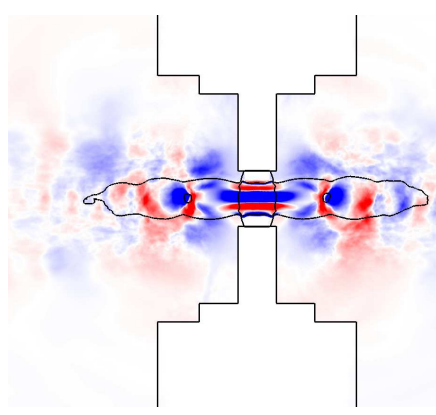

(a) $p^{\prime}$-based POD mode 1

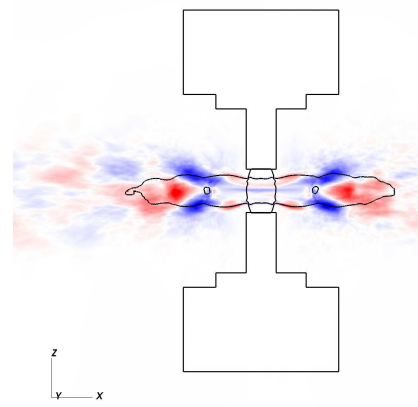

(d) $K^{\prime}$-based POD mode 1

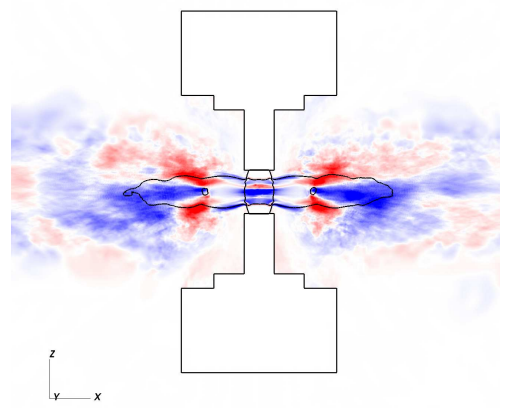

(g) $s^{\prime}$-based POD mode 1

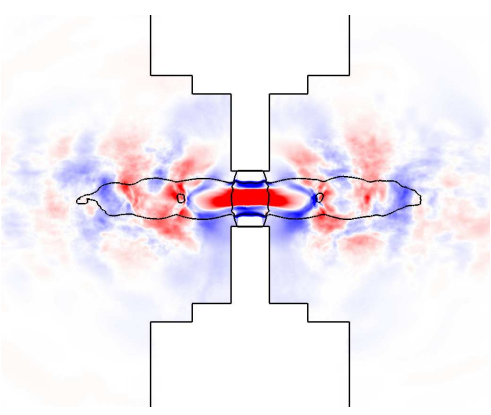

(b) $p^{\prime}$-based POD mode 2

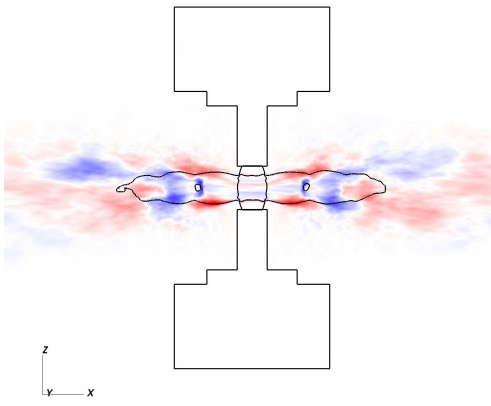

(e) $K^{\prime}$-based POD mode 2

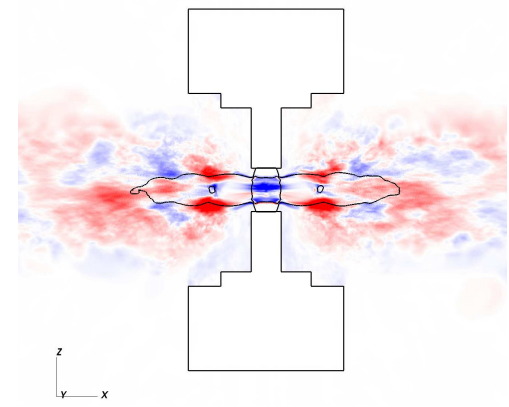

(h) $s^{\prime}$-based POD mode 2

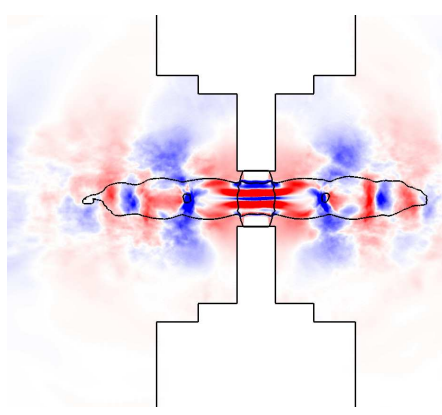

(c) $p^{\prime}$-based POD mode 3

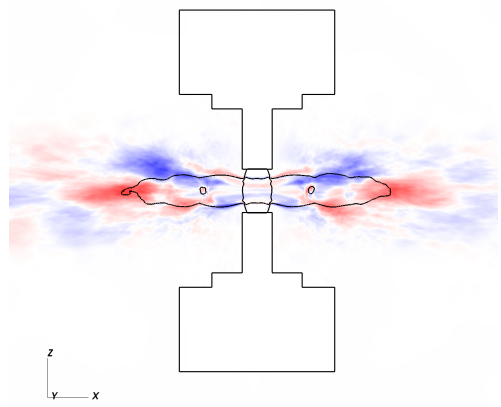

(f) $K^{\prime}$-based POD mode 3

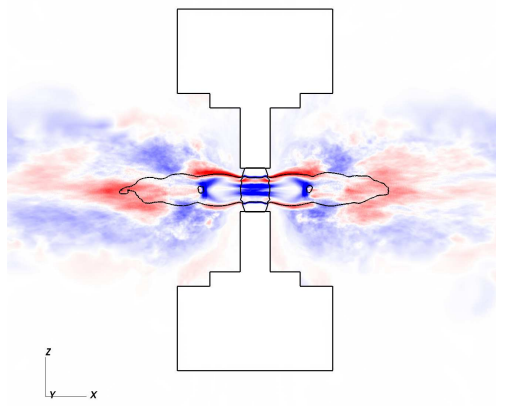

(i) $s^{\prime}$-based POD mode 3

Figure 14. Colored contours of the first three most energetic POD modes using an energy norm based on (a-c) pressure, (d-f) turbulent kinetic energy, and (g-i) entropy fluctuation term.

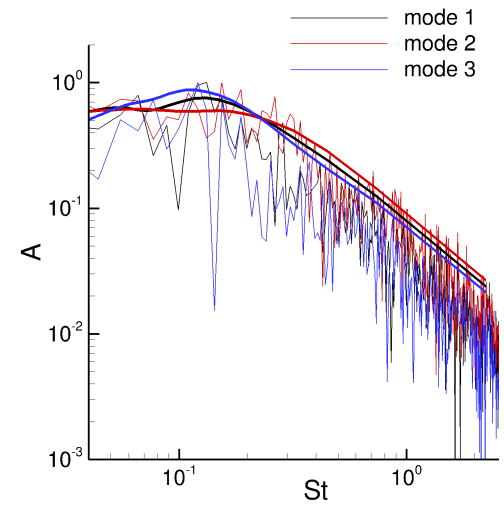

(a) $p^{\prime}$-based

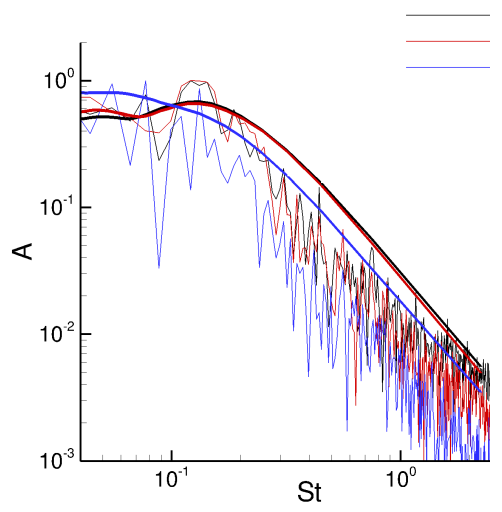

(b) $K^{\prime}$-based

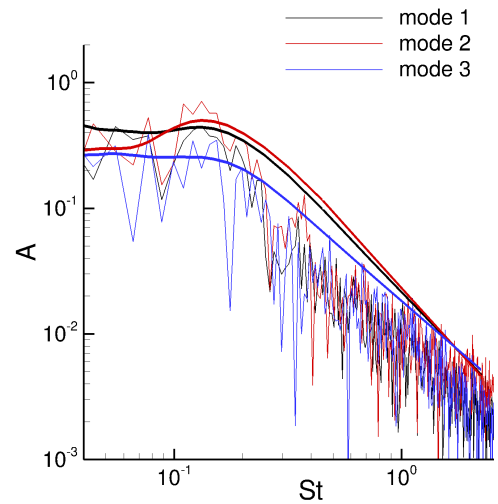

(c) $s^{\prime}$-based

Figure 15. Fourier and DWT pseudo spectrum of POD time-coefficients of three most energetic POD modes using an energy norm based on (a) pressure, (b) turbulent kinetic energy, and (c) entropy fluctuation term.

\section{Noise Source Identification}

One of the key objectives of this work is to identify the dominant noise sources and uncover the relevant physical mechanisms responsible for noise generation. To investigate noise generation mechanisms, the 
dynamics of the unsteady flow field discussed in section III need to be connected to the radiated sound field. Simultaneous visualizations of the instantaneous flow and sound fields can provide an idea on specific events that generate noise, see for example. ${ }^{33,40,41}$ In the current study, we employ a more direct approach by using the causality method, which relies on computing cross-correlations between flow quantities inside the four-jet impingement flow field and the radiated acoustic pressure outside the non-linear flow regime. An attractive feature of this method is that the effects of scattering, absorption and refraction on sound radiation are automatically included by simultaneously extracting information from both the flow and acoustic fields. The present work is based upon the causality idea proposed in earlier works by. ${ }^{11-13}$ Computing the correlation between turbulent fluctuations inside the jet and the radiated noise in the far field is the most direct and unambiguous way of identifying the relevant noise sources. The current theoretical framework is based on the acoustic analogy employed in Lighthill's equation (6). It is well-known that the decomposition proposed in Lighthill's analogy is non-unique. Flow-acoustic interactions are generally not distinguished from true acoustic sources. ${ }^{9}$ However, more elaborate formulations aimed at extending the acoustic analogy to account for the flow-acoustic interactions sacrifice the inherent simplicity of Lighthill's original approach. In the current paper, flow-acoustic interaction is not explicitly accounted for and the noise sources shall be interpreted in terms of Lighthill's sources. These source term, however, contains fluid dynamics that do not follow the homogeneous-medium second-order wave equation. Because flow-acoustic interactions have been demonstrated to be important in shear layers, the results need to be interpreted carefully. ${ }^{42,43}$

Lighthill's equation is defined as

$$
\frac{\partial \rho}{\partial t}-a_{\infty}^{2} \nabla^{2} \rho=\frac{\partial^{2} T_{i j}}{\partial x_{i} \partial x_{j}}
$$

where the right-hand-side term contains Lighthill's stress tensor $T_{i j}=\rho u_{i} u_{j}+\delta_{i j}\left(p-a_{\infty}^{2} \rho\right)$ absent the effect of viscosity, $u_{i}$ is the unsteady velocity field, and $p$ is the unsteady pressure field. In the current discussion, it is assumed that the viscous effects are negligible for the noise generation as well as feedback from the acoustic field to the source. It is important to remember that Lighthill's equation is simply a reformulation of the mass and momentum equations and is valid for every solution that

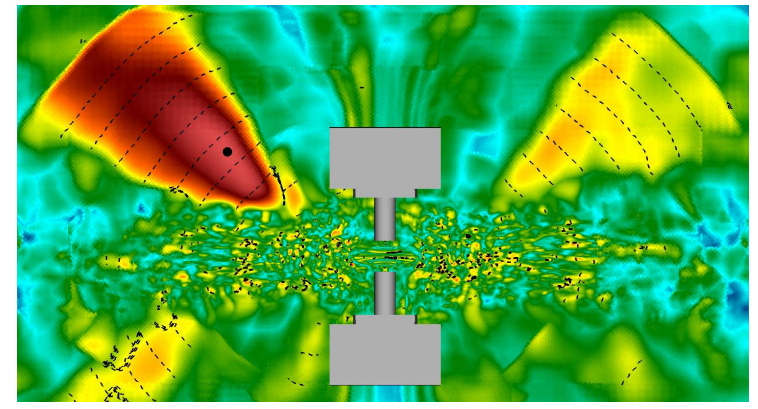

Figure 16. Normalized cross-correlation of acoustic pressure at sample point marked with solid circle (•) and pressure on the sampling plane. obeys these equations. Lighthill's equation cannot distinguish between radiating and non-radiating disturbances. Theoretically, the relationship $a_{\infty}<\omega / \alpha$ can be used to separate the radiating and non-radiating parts, where $\omega$ is the angular frequency and $\alpha$ is the spatial wave number. In the current approach, we do not utilize this relationship explicitly and pursue a slightly different route to identify the radiating part of the solution. We follow the idea that multiplying with the acoustic pressure fluctuations in the far field acts simply as a filter operation, because sampling points located in the far field only sense the radiated part of disturbances. ${ }^{7}$

The most relevant parts of the causality approach are presented below. The theoretical foundation of our noise source identification strategy is laid out in more detail in Brehm et al. ${ }^{4}$ The right-hand side of equation (6) can be regarded as a forcing term to the wave equation. Acoustically, the source terms represent a distribution of quadrupoles embedded in an ambient medium whereby flow discontinuities are not explicitly contemplated. The desired solution at a far-field point is derived in terms of an integral equation after manipulating equation (6) and utilizing the time-domain free-space Green's function.

$$
\begin{aligned}
p^{\prime}\left(\boldsymbol{x}_{f}, t\right) & \approx \frac{x_{i} x_{j}}{4 \pi a_{\infty}^{2} r^{2}} \frac{\partial^{2}}{\partial t^{2}} \int_{V} \frac{T_{i j}\left(\boldsymbol{x}_{s}, t^{\prime}\right)}{\left|\boldsymbol{x}_{f}-\boldsymbol{x}_{s}\right|} d V+\frac{1}{4 \pi} \frac{\partial}{\partial t} \int_{S} \frac{\rho u_{i}}{\left|\boldsymbol{x}_{f}-\boldsymbol{x}_{s}\right|} n_{i} d S \\
& +\frac{x_{j}}{4 \pi r} \frac{\partial}{\partial t} \int_{S} \frac{\left(\delta_{i j} p+\rho u_{i} u_{j}\right)}{\left|\boldsymbol{x}_{f}-\boldsymbol{x}_{s}\right|} n_{i} d S .
\end{aligned}
$$

In contrast to the jet impingement problem discussed in Brehm et al. ${ }^{4}$ the contributions from the surface integrals in equation (7) are negligible except of scattering effects. Following the analysis in Proudman ${ }^{44}$ as more recently revisited by Panda et al.. ${ }^{7}$ we introduce the scalar component of the stress tensor $T_{r}$ that represent longitudinal quadrupoles. The scalar quantity is the fluctuating stress tensor component $T_{r}$ in the direction of the source point to the far-field observer location, i.e., $\boldsymbol{r}=\boldsymbol{x}_{f}-\boldsymbol{x}_{s}$. The acoustic intensity 
at a far-field point is finally obtained by multiplying equation (7) with the far-field acoustic pressure and computing the acoustic intensity,

$$
\begin{aligned}
\left\langle p^{\prime}, p^{\prime}\right\rangle\left(\boldsymbol{x}_{f}, \tau\right) & =\left\langle\frac{1}{4 \pi a_{0}^{2} \boldsymbol{r}} \int_{V} \frac{\partial^{2}}{\partial t^{\prime 2}} T_{r}\left(\boldsymbol{x}_{s}, t^{\prime}+\tau\right) d V, p^{\prime}\left(\boldsymbol{x}_{f}, t\right)\right\rangle \\
& =\frac{1}{4 \pi a_{0}^{2} \boldsymbol{r}} \int_{V} \frac{\partial^{2}}{\partial \tau^{2}} C_{T_{r}, p^{\prime}}\left(\boldsymbol{x}_{s}, \boldsymbol{x}_{f}, \tau\right) d V,
\end{aligned}
$$

where the correlation function $C_{T_{r}, p^{\prime}}$ is evaluated after shifting the near field data $T_{r}$ by the propagation time or time delay $\tau$. Equation (8) provides an elegant way of linking the near field dynamics to the farfield acoustic pressure fluctuations. Applying the cross-correlation is extremely powerful since it naturally separates the hydrodynamic and acoustic fluctuations. ${ }^{7}$ To avoid the computation of the second derivatives in time, we apply a temporal Fourier transform to equation (8) and find

$$
P S D_{p^{\prime}}\left(\boldsymbol{x}_{f}, f\right)=-\frac{\pi f^{2}}{r a_{\infty}^{2}} \int_{V} C S D_{T_{r}, p^{\prime}}\left(\boldsymbol{x}_{f}, \boldsymbol{x}_{s}, f\right) d V
$$

where the auto-correlation function of $p^{\prime}$ is transformed to the power spectral density $P S D_{p^{\prime}}$ and the crosscorrelation function between $T_{r}$ and $p^{\prime}$ turns into the cross-spectral density $C S D_{T_{r}, p^{\prime}}$.

Figure 16 shows contours of the peak normalized cross-correlation function $C_{p^{\prime}, p^{\prime}}^{0}$ of the acoustic pressure at a sample point (solid circle) with the unsteady pressure in the $(x, y)$-sampling plane (superscript 0 denotes the normalization). The dashed lines mark the time-delay of the cross-correlation. An approximate location of the noise source can be obtained by tracing back the contour lines of time-delay to the region of high fluctuation intensity, where the normalized cross-correlation function has small values. Panda et al. ${ }^{45}$ pointed out that simply utilizing normalized correlations may lead to an incorrect interpretation of the noise source distribution because it does not provide information about the fluctuation intensity. Here, we follow the physical expectation that by employing non-normalized correlation functions, the strongest fluctuations can be differentiated as the source and the weaker fluctuations as the effect. Hence, the normalized crosscorrelation function $C^{0}$ provide valuable information about the interplay of vortical flow structures with the unsteady shocks, which leads to significant hydrodynamic pressure fluctuations and the generation of acoustic waves. When employing this approach for the jet impingement problem, ${ }^{5}$ it was demonstrated that it is possible to identify flow features that are relevant for the noise generation process by utilizing normalized cross-correlation functions. In Brehm et al., ${ }^{4}$ large peak correlations of $C_{p^{\prime}, V_{r}^{\prime}}^{0}$ with an observer point at $\theta=120^{\circ}$ were obtained in the potential core region of the jet upstream of the impingement location. It was conjectured that these strong correlations are associated with large-scale coherent flow structures that can be observed in the shear-layer of the jet. These coherent flow structures interact with the solid wall and the oblique shock forming above the impingement plate. The frequency signature of these flow features is imprinted on the acoustic waves predominantly propagating in $\theta=120^{\circ}$ to jet axis. In the present flow field, the normalized cross-correlation functions, $C_{p^{\prime}, V_{r}^{\prime}}^{0}$ and $C_{p^{\prime}, s^{\prime}}^{0}$, did not display large peak values inside the source region similar to $C_{p^{\prime}, p^{\prime}}^{0}$ shown in figure 16 . Hence, by employing normalized crosscorrelation functions we are not able to identify coherent flow features that play an important role in the noise generation process. As discussed in Panda et al. ${ }^{45}$ the acoustic source distribution can only be identified by employing non-normalized cross-correlation functions.

Thus, next non-normalized cross-correlation functions are used to localize the acoustic source distribution in the flow field. Figure 17 shows the non-normalized cross-correlation functions of acoustic pressure at sample point with pressure fluctuations and components of the Lighthill's stress tensor in the $(x, y)$-sampling plane. $C_{p^{\prime}, p^{\prime}}$ displays large amplitudes in the four-jet impingement region and around the Mach disk of the first shock cell. The cross-correlation function $C_{p^{\prime}, V_{r}^{\prime}}$ picks up shear-noise that correlates well with acoustic pressure at the sample point. A significant amount of shock noise is being generated at the Mach disk of the first shock cell based on $C_{p^{\prime}, p^{\prime}}$. Furthermore, strong cross-correlations between the entropy fluctuation term and acoustic pressure can be observed in the center of the four-jet impingement region. Both cross-correlation functions, $C_{p^{\prime}, V_{r}^{\prime}}$ and $C_{p^{\prime}, V_{s}^{\prime}}$, display comparable amplitudes over a significant spatial extent and appear to reveal an important property of the noise generation process. 


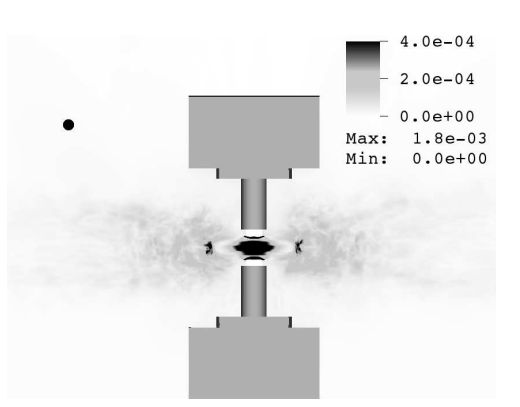

(a) $C_{p^{\prime}, p^{\prime}}$

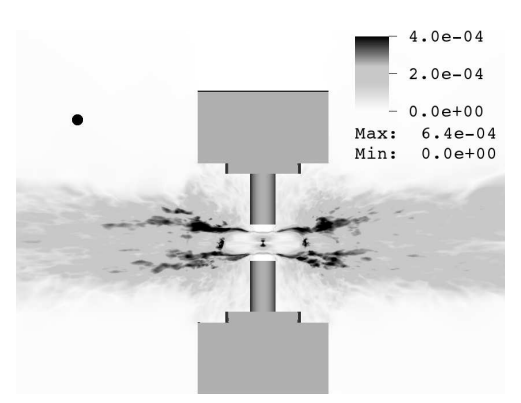

(b) $C_{p^{\prime}, V_{r}^{\prime}}$

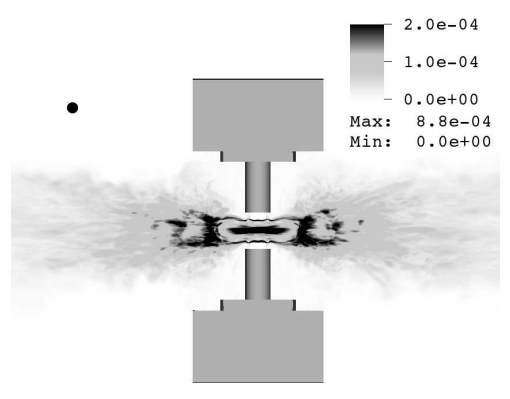

(c) $C_{p^{\prime}, s^{\prime}}$

Figure 17. Non-normalized cross-correlation of acoustic pressure at sample point (•) with pressure fluctuations and components of the Lighthill's stress tensor on the $(x, y)$-sampling plane.

\section{Development of a Reduced-Order Linear Acoustic Model}

In the following discussion, a linear acoustic model of the FJID is constructed based on the findings in the previous analysis. The key ingredient is the utilization of an in-house linear acoustic scattering code that is part of the LAVA framework. ${ }^{14}$ The scattering code is able to perform acoustic calculations with far less computational resources than when utilizing the compressible Navier-Stokes solver. This is especially advantageous when propagating sound waves to far-field locations that are several characteristic length scales away from the source region. The linear acoustic scattering code is based on solving the linear Helmholtz boundary value problem in the frequency domain using a highly scalable equivalent source method (see Ochmann ${ }^{46}$ ). To solve the boundary value problem $N_{M}$ monopole sources are placed inside the scattering surface that contains $N_{S}>N_{M}$ surface elements (here, triangles). Utilizing the free-space Green's function for a monopole source the overall acoustic pressure field is obtained following the superposition principle. The strength of the monopoles is obtained by solving an over-determined system of equation that accounts for the boundary conditions at each element of the scattering surface. It is assumed that the incident pressure field is either available on a radiating surface where the unsteady data is extracted from high-fidelity CFD simulations (permeable surfaces are used that encompass the source region) or an equivalent acoustic model can be used to effectively mimic the noise being generated in the source region. In the present work, we follow the latter route by creating a linear acoustic model that is able to capture the main features of the acoustic sound field. This approach was taken because the understanding

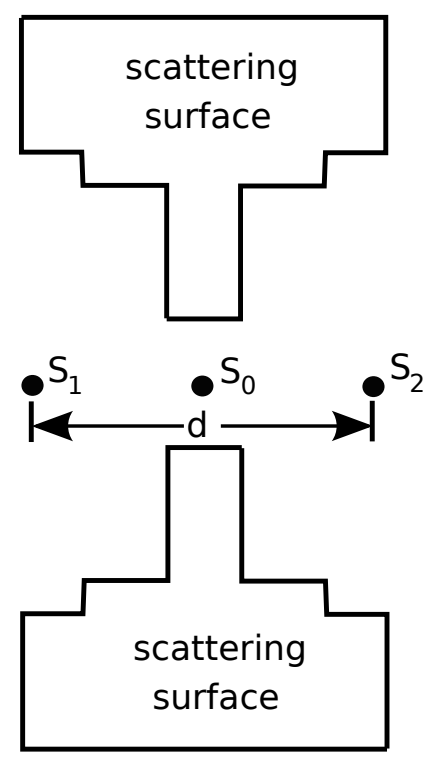

Figure 18. Preliminary linear acoustic model containing three sources, $S_{0}, S_{1}$ and $S_{2}$, placed in the vicinity of the FJID scattering surface. of the noise generation mechanisms in the FJID obtained from the previous analyses can be directly tested by comparison with available CFD data in the acoustic near-field. When deploying three of the reduced-order linear acoustic FJID models inside the engine nacelle to simulate the BENS experiment it must be ensured that the three FJID noise sources are temporally uncorrelated. In prior research studies, ${ }^{1}$ the FJID was modeled as a simple broadband monopole noise source. In this section, we describe a more sophisticated FJID model. The previous analysis of the ILES simulations provides valuable information about the noise generation process in the FJID. Three important noise source regions were identified, i.e., the jet impingement region, the shear-layers of the primary jets and the end of the first shock diamond structure. Most importantly, it can be noted that the surface of the FJID itself provides a scattering field that needs to be considered in the model. To model the acoustic characteristics of the FJID we used a simple linear acoustic model. Figure 18 displays the setup of the linear acoustic model. Three monopole sources, denoted by $S_{0}, S_{1}$ and $S_{2}$, were placed in the vicinity of the FJID scattering surface. The monopole source $S_{0}$ is supposed to capture the dynamics in the high pressure four jet impingement region. The objective of $S_{1}$ and $S_{2}$ is to primarily model the unsteadiness around the Mach disk of the first shock cell and account indirectly for the accumulative noise generation in the shear-layers of the primary jets. It is clear that this model is overly simplified and does not account for the most basic features of the noise generation 
process. For example, if Mach radiation from large-scale coherent flow structures is a key noise generation mechanism a single monopole placed on the center-line of the primary jet would not be able to reproduce the actual directivity pattern. Nevertheless, it appears that this model is able to capture some of the key features of the acoustic pressure field. In the future, we are planning on refining this reduced-order model to improve the model of the noise generation mechanisms and possibly account for flow-acoustic interactions. In this study, we focus on results for $S t \approx 0.2$ (or $f=31.5 \mathrm{kHz}$ ) since this frequency corresponds to the peak frequency (roughly $1.8 \mathrm{kHz}$ for full scale of the HWB) for turbomachinery noise. ${ }^{2}$

Since the FJID produces a broadband noise field it may be important to consider the characteristics of broadband noise sources in the linear acoustic model. In the current investigation, monochromatic and broadband (white) noise sources are considered. The assumption of white noise is justified because we aim at modeling broadband noise in one-third octave-bands (as in the experimental study by Hutcheson et $_{\text {al. }}{ }^{2}$ ) and our CFD results show that the amplitude is fairly constant within the one-third octave-band centered around $S t=0.2$. When comparing monochromatic noise and broadband noise it is important to establish an equivalent monochromatic noise source amplitude. Considering the root-mean-square pressure field of the signal a simple relationship between the source strengths of single and multiple frequency signal can be obtained.

$$
A_{\text {mono }}=\sum_{n=1}^{N} A_{b b, n}^{2}
$$

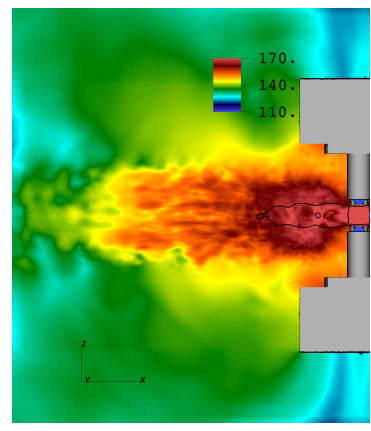

(a) CFD

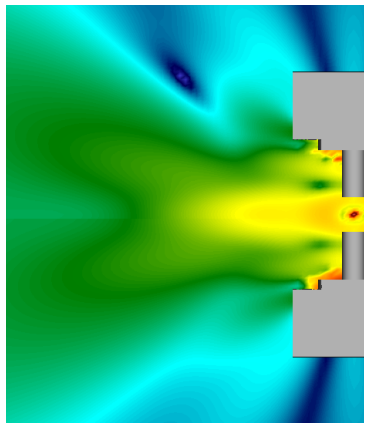

(b) $S_{0}=1$

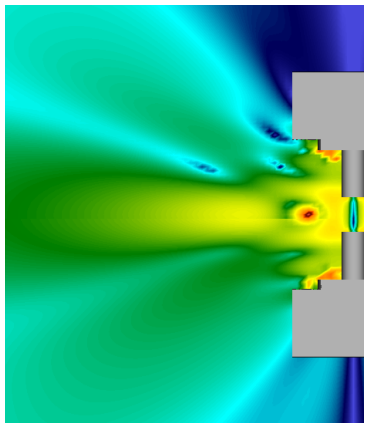

(c) $S_{0}=0, S_{1}=S_{2}=0.5$

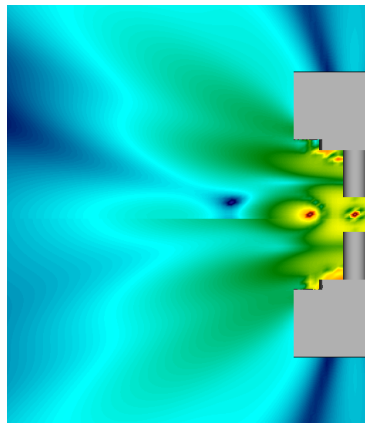

(d) $S_{0}=S_{1}=S_{2}=1 / 3$

Figure 19. Sound pressure level in sampling plane for (a) CFD, and (b-d) different acoustic models

Figures 19a-d show the SPL levels for CFD data and different linear acoustic models. All results shown here were obtained for a frequency of $S t=0.2$. The SPL levels from the CFD data were computed in one-third octave-bands with a center frequency of $S t=0.2$. No amplitude values are provided for the results of the linear acoustic scattering code because the amplitudes of the sources can be scaled due to the linearity of the problem. By comparing the linear acoustic results with acoustic data extracted from our CFD runs the amplitude of the sources can later be adjusted. For the linear acoustic model, two solutions are shown for the narrow band solution $(S t=0.2)$ on the top half and the broadband solution $(S t \in[0.179,0.225])$ on the bottom half. The narrow band and broadband solutions are almost identical, which is not surprising with $S t /(0.5 \Delta S t)>8$. Consequently, from this point forward the narrow band solutions are utilized. For lower frequencies and different noise source configurations, this assumption needs to be re-evaluated because the broadband results can be significantly different from narrow-band results.

The wave propagation pattern can be visualized by plotting the phase of the complex Fourier coefficients in the sampling plane. Figures 20a-d show the phase plots for CFD and different linear acoustic models. The phase shift on the top and bottom of the four-jet impingement device in the CFD data suggests that the acoustic field has dipole characteristics.

The phase relationship that one should impose among the noise sources to produce a realistic sound field is unclear. As one option, we try introducing a random phase relationship to completely decorrelate the sources. The overall solution is obtained as an average over these solutions as shown in figures 21 for $S_{0}=S_{1}=S_{2}, 2 S_{0}=S_{1}=S_{2}$, and $S_{0}=S_{1}+S 2$. In these solutions it was assumed that $S_{1}$ and $S_{2}$ have a zero phase relationship, which is not necessarily the case given the coupled flapping motion of the jets on each side. The POD analysis and the causality method showed, however, that a perfect symmetry between both sides exists with respect to the large-scale motion inside the source region. Furthermore, the time-delay in the cross-correlation functions to obtain a peak correlation between the left and right side of the FJID 


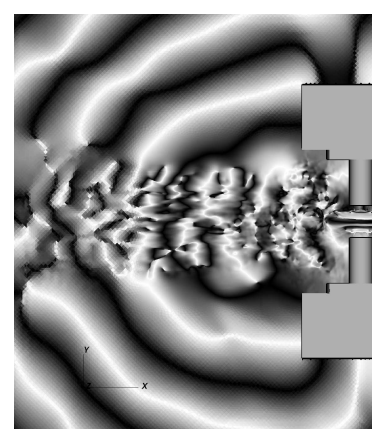

(a) CFD

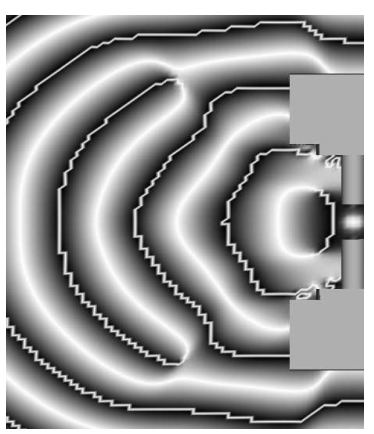

(b) $S_{0}=1$

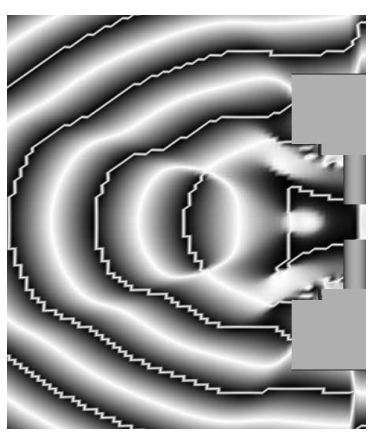

(c) $S_{0}=0, S_{1}=S_{2}=0.5$

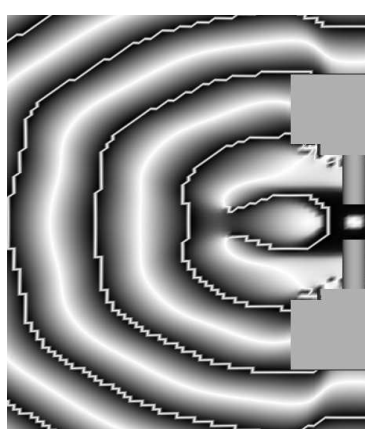

(d) $S_{0}=S_{1}=S_{2}=1 / 3$

Figure 20. Phase plots in sampling plane for (a) CFD, and (b-d) different acoustic models

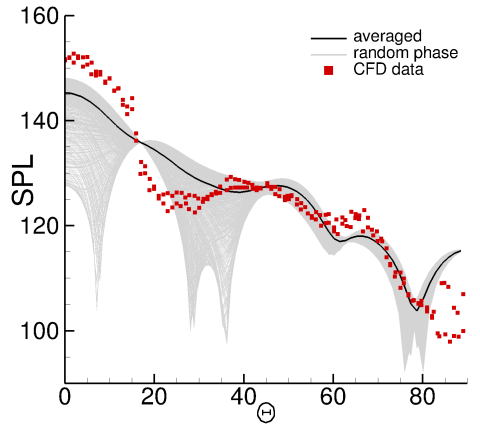

(a) $S_{0}=S_{1}=S_{2}$

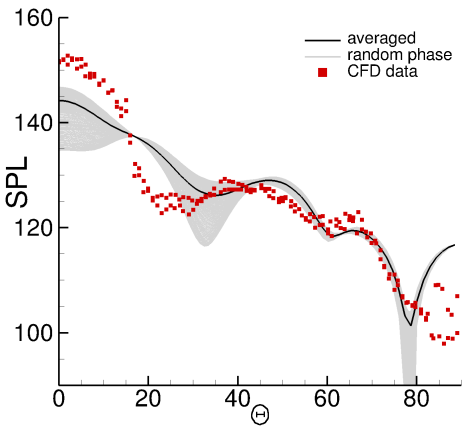

(b) $2 S_{0}=S_{1}=S_{2}$

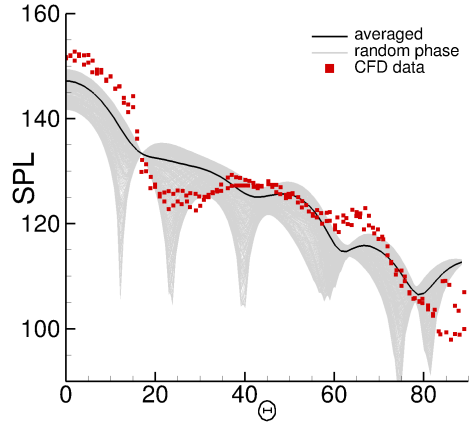

(c) $S_{0}=S_{1}+S 2$

Figure 21. Different acoustic models with randomized phases between $S_{0}$ and $S_{1}=S_{2}$.

is $\tau=0$. Considering the available data set, it appears that the linear acoustic model reproduces the CFD data best when using a $S_{0}=S_{1}=S_{2}$ with $d / D=5.7$. Note that the jet-region pressure levels shown in the reduced-order model in figure 21(b-d) under-predict the levels shown in the CFD data (figure 21a) due to the presence of significant hydrodynamic pressure fluctuations in the unsteady jets. Hence, a meaningful comparison of the SPL values for $\Theta \in\left[0,20^{\circ}\right]$ is not possible at this point.

\section{Validation of Linear Acoustic FJID Model for BENS Experiment}

The final part of this paper is concerned with placing three instances of the linear acoustic FJID model developed in section VII in an engine nacelle as discussed in Hutcheson et al. ${ }^{2}$ Figure 22a schematically illustrates the computational setup used in this study. In the present study, only the no-flow case $\left(M_{\infty}=0\right)$ is considered where the engine inlet was capped. The setup is similar to the one discussed in Tinetti and Dunn $^{1}$ but includes some additional geometry, such as plug and bracket. Each of the blue spheres in figure 22a represents a linear acoustic FJID model. For the current analysis, $1 \times 10^{6}$ triangles and $60 \times 10^{3}$ monopole sources are placed inside the scattering surfaces as displayed in figure $22 \mathrm{~b}$. The sensitivity with respect to various modeling parameters in the acoustic scattering analysis employing the ESM were studied by varying (i) the monopole offset distance to the scattering surface, (ii) the number of equivalent monopole sources, (iii) the resolution of triangles on the scattering surface, and (iv) the number of sample sets with different phases between the three FJID acoustic models.

The scattering solution procedure proceeds in the following three steps:

1. Obtain linear acoustic scattering solution employing the equivalent monopole source method separately for each FJID

2. Apply random $N=1000$ phases to each of the three solutions and compute phase-averaged solutions

3. Superimpose the three phase-averaged solutions and extract sound pressure levels on sampling points 


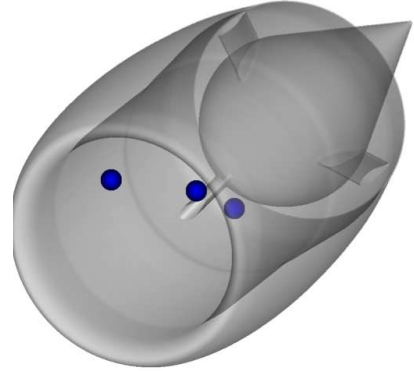

(a)

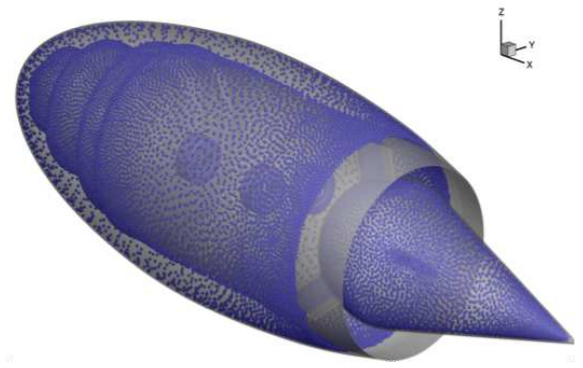

(b)

Figure 22. (a) BENS linear acoustic scattering simulation setup and (b) equivalent monopole sources inside engine nacelle

Step 2 is necessary in order to ensure that the three FJID models are statistically uncorrelated. The random phases were generated with conventional Latin hypercube sampling. It is essentially the same approach as previously discussed in section VII.

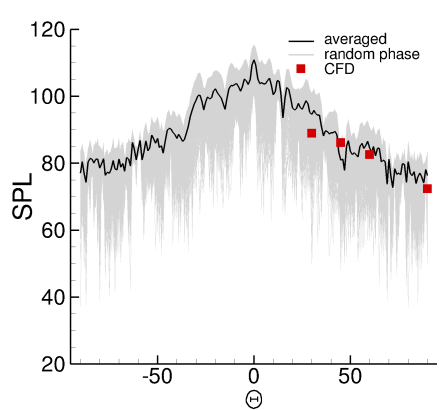

(a) $S_{0}=S_{1}=S_{2} \&$ FJID surface

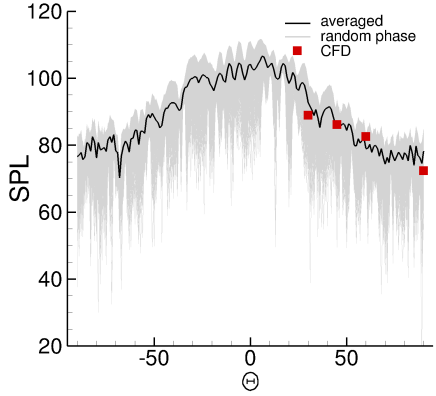

(b) $S_{0} \&$ FJID surface

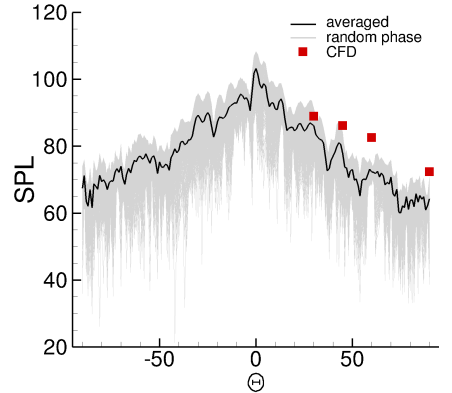

(c) $S_{0}$ only

Figure 23. Sound pressure level in sampling plane for (a) CFD, and (b-d) different acoustic models

Figures 23a-c provides a comparison of the experimental data with acoustic scattering results. Three types of linear acoustic FJID models were utilized: (a) $2 S_{0}=S_{1}+S_{2}$ with FJID scattering surface, (b) $S_{0}$ with FJID scattering surface, and (c) $S_{0}$ without FJID scattering surface. For models (a) and (b) fair comparison with experimental data is achieved. The results demonstrate that including the FJID scattering surface appears to be important to match the experimental data. When including the FJID models inside the engine nacelle the sensitivity with respect to the particularities of the noise source generation discussed above are significantly reduced. This is not surprising for this application since the acoustic pressure waves can only escape through a very narrow gap in the engine exit.

\section{Conclusions}

The main objective of this paper was to study the underlying physics of the four-jet impingement problem that spawn acoustically radiating pressure waves. The grid convergence study considering the spectral energy of pressure ensured sufficient grid resolution for resolving the flow physics relevant for the noise generation process of the FJID with the current CFD simulation approach. Proper orthogonal decomposition was used to identify the most energetic flow features in the source region. The most energetic $p^{\prime}$-based POD modes display large amplitudes inside the jet impingement region. These large pressure fluctuations are associated with the sporadic appearance of vortex tubes gravitating towards the center of the impingement region. These vortex tubes originate at the inception of the four diagonally ejected secondary jets and slowly gravitate towards the center of the high pressure region. The most energetic $K^{\prime}$-based POD modes mainly capture the dynamics in the shear layers and the interaction of the shear layer with the shock cells. The most energetic $s^{\prime}$-based POD modes display a strong coherence between the unsteadiness inside the impingement region and the unsteady motion of the first shock diamond structure of the two primary (deflected) jets. 
The frequency signature of the POD modes is broadband in nature, as it is for the $p^{\prime}$-based POD modes. However, most energetic $K^{\prime}$-based and $s^{\prime}$-based POD modes contain a broadband peak around $S t=0.2$. By employing the causality method, it was possible to link different components of the Lighthill's stress tensor to the acoustic pressure in the far-field. Two key noise generation mechanisms were identified: (i) shear layer interaction with the first Mach diamond structure, and (ii) large entropy fluctuations within the impingement region that is coupled to unsteady shock motion inside the two deflected jets.

The second part of the paper was concerned with constructing a reduced-order linear acoustic model that is able to capture the main characteristics of the acoustic pressure field of the FJID. Based on the noise source analysis, it appears that the acoustic pressure field of the FJID can be modeled reasonably well with a simple linear acoustic model considering three monopole sources. The single monopole source in the center of the FJID is supposed to capture the dynamics in the jet impingement region. The two monopole sources separated by a distance $d$ model the unsteadiness around the Mach disk of the first shock cell and indirectly account for the accumulative noise generated in the shear-layers of the primary jets. The linear acoustic analysis demonstrated that it is important to include the scattering surface of the FJID itself to be able to match the LES data. In a first step towards modeling the Broadband Engine Noise Simulator (BENS) experiment studied by Hutcheson et al., ${ }^{2}$ three linear acoustic FJID models were placed inside a front capped engine nacelle. With this preliminary model, fair agreement with experimental data was obtained. The CAA results, however, also demonstrate that the acoustic predictions are not very sensitive to some of the details of the linear acoustic FJID model. This may be explained by considering that the pressure waves can only escape through a small opening at the engine exit. An important achievement of this work is that the current approach for modeling the acoustics of the BENS experiment provides a truly predictive capability because it is solely based on our CFD/CAA predictive capabilities of the four-jet impingement problem.

\section{Appendix A: Computation of POD Modes}

The POD eigenmodes are then computed as

$$
\vec{\psi}^{(n)}(\vec{x})=\sum_{i=0}^{N} r_{i}^{(n)} \vec{q}\left(\vec{x}, t_{i}\right),
$$

where the subscript $i$ indicates the time-step $(i=0$ to $N), \vec{q}\left(\vec{x}, t_{i}\right)$ is the vector of flow quantities at timestep $i$, and $r_{i}^{(n)}$ are individual elements of $\vec{r}^{(n)}$. The right eigenvectors, $\vec{r}^{(n)}$, are solutions to the algebraic eigenvalue problem:

$$
\mathbf{C} \vec{r}^{(n)}=\lambda^{(n)} \vec{r}^{(n)},
$$

where the correlation tensor $\mathbf{C}$ is defined in index notation as

$$
C_{i j}=\frac{1}{N+1}\left[\vec{q}\left(\vec{x}, t_{i}\right), \vec{q}\left(\vec{x}, t_{j}\right)\right] .
$$

Here, the brackets denote the inner product $[\vec{q}, \vec{q}]=\int_{\Omega}\left(\sum_{k=0}^{N_{q}} \omega_{k} q_{k}^{2}\right) d \vec{x}$ (see Equation (4)). The eigenfunctions are normalized with their corresponding eigenvalues $\left[\vec{\psi}^{(n)}, \vec{\psi}^{\left(n^{\prime}\right)}\right]=\delta_{n n^{\prime}} \lambda^{(n)}$, and the time coefficients are computed as

$$
a^{(n)}(t)=\frac{1}{\lambda^{(n)}}\left[\vec{q}(\vec{x}, t), \vec{\psi}^{(n)}(\vec{x})\right] .
$$

The flow field can be reconstructed/recovered from the eigenmodes and time coefficients:

$$
\vec{q}(\vec{x}, t) \approx \sum_{n=0}^{I} a^{(n)}(t) \vec{\psi}^{(n)}(\vec{x}) .
$$

The POD modes are orthogonal to each other and sorted by their respective energy norm (see Equation (4)) contents, whereby the drop-off in mode energy toward the higher mode numbers is typically significant. Therefore, a small number of modes, I, will suffice for capturing a large percentage of the flow's energy defined in Equation (4). This is particularly the case when the flow dynamics are governed by large energetic structures of organized (or coherent) fluid motion (such as in pure two-dimensional simulations). 


\section{Acknowledgements}

The authors would like to thank Russell Thomas, Christopher Bahr, and Casey Burley from NASA Langley Research Center (LaRC) and Kevin James from NASA Ames Research Center (ARC) for their valuable contributions to this work. The authors also would like to thank Ted Manning and Emre Sozer from NASA Ames Research Center (ARC) for their excellent feedback during the ARC review process. This work was funded by NASA's Environmentially Responsible Aviation (ERA) project and computer time was provided by NASA Advanced Supercomputing (NAS) facilities at ARC.

\section{References}

${ }^{1}$ A., T. and M., D., "Scattering of Simulated Broadband Noise by Conventional and Next Generation Aircraft," 18th AIAA/CEAS Aeroacoustics Conference, Colorado Springs, CO, June 2012, AIAA 2012-2075.

${ }^{2}$ Hutcheson, F. V., Brooks, T. F., Burley, C. L., Bahr, C. J., Stead, D. J., and Pope, D. S., "Shielding of Turbomachinery Broadband Noise from a Hybrid Wing Body Aircraft Configuration," June 2014, AIAA 2014-2624.

${ }^{3}$ Clark, L. R. and Gerhold, C. H., "Inlet noise reduction by shielding for the blended-wing-body airplane," AIAA paper, , No. 99-1937, 1999, pp. 10-12.

${ }^{4}$ Brehm, C., Hader, C., and Fasel, H., "A locally stabilized immersed boundary method for the compressible NavierStokes equations," JCP, 2015.

${ }^{5}$ Brehm, C., Sozer, E., Moini-Yekta, S., Housman, J., Barad, M., Kiris, C., Vu, B., and Parlier, C., "Computational Prediction of Pressure Environment in the Flame Trench," AIAA Paper, June 24-27, San Diego, CA, 2013.

${ }^{6}$ Panda, J. and Seasholtz, R., "Experimental investigation of density fluctuations in high-speed jets and correlation with generated noise," Journal of Fluid Mechanics, Vol. 450, 2002, pp. 97-130.

${ }^{7}$ Panda, J., Seasholtz, R., and Elam, K., "Investigation of noise sources in high-speed jets via correlation measurements," Journal of Fluid Mechanics, Vol. 537, 2005, pp. 349-385.

${ }^{8}$ Tam, C. K., Viswanathan, K., Ahuja, K., and Panda, J., "The sources of jet noise: experimental evidence," Journal of Fluid Mechanics, Vol. 615, 2008, pp. 253-292.

${ }^{9}$ Freund, J., "Noise sources in a low-Reynolds-number turbulent jet at Mach 0.9," Journal of Fluid Mechanics, Vol. 438, 2001, pp. 277-305.

${ }^{10}$ Bogey, C. and Bailly, C., "An analysis of the correlations between the turbulent flow and the sound pressure fields of subsonic jets," Journal of Fluid Mechanics, Vol. 583, 2007, pp. 71-97.

${ }^{11}$ Siddon, T., "Noise source diagnostics using causality correlations," AGARD CP, Vol. 131, 1973, pp. 7-1.

${ }^{12}$ Seiner, J. M., "The distribution of jet source strength intensity by means of a direct correlation technique," 1974.

${ }^{13}$ Rackl, R. and Siddon, T., "Causality correlation analysis of flow noise with fluid dilatation as source fluctuation," The Journal of the Acoustical Society of America, Vol. 65, No. 5, 1979, pp. 1147-1155.

${ }^{14}$ Kiris, C., Barad, M., Housman, J., Sozer, E., Brehm, C., and Moini-Yekta, S., "The LAVA Computational Fluid Dynamics Solver," AIAA Paper 2014-0070, Jan 13-17, National Harbor, Maryland, 2014.

${ }^{15}$ Berger, M. J. and Colella, P., "Local Adaptive Mesh Refinement for Shock Hydrodynamics," J. Comput. Phys., Vol. 82, No. 1, May 1989, pp. 64-84.

${ }^{16}$ Almgren, A. S., Bell, J. B., Colella, P., Howell, L. H., and Welcome, M. L., "A Conservative Adaptive Projection Method for the Variable Density Incompressible Navier-Stokes Equations," J. Comp. Phys., Vol. 142, 1998, pp. 1-46.

${ }^{17}$ Barad, M. F. and Colella, P., "A Fourth-Order Accurate Local Refinement Method for Poisson's Equation," J. Comp. Phys., Vol. 209, No. 1, October 2005, pp. 1-18.

${ }^{18}$ Barad, M. F., Colella, P., and Schladow, S. G., "An Adaptive Cut-Cell Method for Environmental Fluid Mechanics," Int. J. Numer. Meth. Fluids, Vol. 60, No. 5, 2009, pp. 473-514.

${ }^{19}$ Zhang, Q., Johansen, H., and Colella, P., "A Fourth-Order Accurate Finite-Volume Method with Structured Adaptive Mesh Refinement for Solving the Advection-Diffusion Equation," SIAM Journal on Scientific Computing, Vol. 34, No. 2, 2012, pp. 179-201.

${ }^{20}$ Colella, P., Graves, D. T., Ligocki, T. J., Martin, D. F., Modiano, D., Serafini, D. B., and Straalen, B. V., "Chombo Software Package for AMR Applications - Design Document," unpublished.

${ }^{21}$ Mittal, R., Dong, H., Bozkurttas, M., Najjar, F., Vargas, A., and von Loebbecke, A., "A versatile sharp interface immersed boundary method for incompressible flows with complex boundaries," Journal of Computational Physics, Vol. 227, No. 10, 2008, pp. $4825-4852$.

${ }^{22}$ Brehm, C. and Fasel, H., "A novel concept for the design of immersed interface methods," Journal of Computational Physics, Vol. 242, No. 0, 2013, pp. $234-267$.

${ }^{23}$ Brehm, C., Barad, M., Housman, J., and Kiris, C., "A Comparison of Higher-Order Shock Capturing Schemes Within the LAVA CFD Solver," AIAA Paper 2014-1278, Jan 13-17, National Harbor, Maryland, 2014.

${ }^{24}$ Hutcheson, F., Private communication, 2014.

${ }^{25}$ Shur, M., Spalart, P., and Strelets, M., "Noise Prediction for Increasingly Complex Jets. Part I: Methods and Tests," International Journal of Aeroacoustics, Vol. 4, No. 3, 2005, pp. 213-246.

${ }^{26}$ Shur, M., Spalart, P., and Strelets, M., "Noise Prediction for Increasingly Complex Jets. Part 2: Applications," International Journal of Aeroacoustics, Vol. 4, No. 4, 2005, pp. 247-266.

${ }^{27} \mathrm{Hu}, \mathrm{X}$. and Adams, N., "Scale separation for implicit large eddy simulation," Journal of Computational Physics, Vol. 230, No. 19, 2011, pp. 7240-7249. 
${ }^{28} \mathrm{Hu}$, X., Wang, Q., and Adams, N., "An adaptive central-upwind weighted essentially non-oscillatory scheme," Journal of Computational Physics, Vol. 229, No. 23, 2010, pp. 8952-8965.

${ }^{29}$ Shur, M., Spalart, P., Strelets, M., and Travin, A., "Towards the prediction of noise from jet engines," International Journal of Heat and Fluid Flow, Vol. 24, No. 4, 2003, pp. 551 - 561, Selected Papers from the Fifth International Conference on Engineering Turbulence Modelling and Measurements.

${ }^{30}$ Akamine, M., Nakanishi, Y., Okamoto, K., Teramoto, S., Okunuki, T., and Tsutsumi, S., "Experimental Study on Acoustic Phenomena of Supersonic Jet Impinging on Inclined Flat Plate," 52nd Aerospace Science Meeting, January 2014, AIAA 2014-0879.

${ }^{31}$ Michalke, A., "Survey on jet instability theory," Prog. Aerosp. Sci., Vol. 21(3), 1984, pp. 159-199.

${ }^{32}$ Lew, P., Uzun, A., Blaisdell, G., and Lyrintzis, A., "Effects of Inflow Forcing on Jet Noise Using 3-D Large Eddy Simulation," AIAA Paper No. 2004-0516, January, 2004.

${ }^{33}$ Bogey, C. and Bailly, C., "LES of High Reynolds, High Subsonic Jet: Effects of the Inflow Conditions on Flow and Noise," AIAA Paper No. 2003-3170, May, 2003.

${ }^{34}$ Lumley, J., "The structure of inhomogeneous turbulent flows," Atm. Turb. And Radio Wave Prop., Nauka, Moscow and Toulouse, France, eds. Yaglom and Tatarsky, 1967, pp. 166-178.

${ }^{35}$ Freund, J. B. and Colonius, T., "POD Analysis of Sound Generation by a Turbulent Jet," AIAA 2002-0072, 40th AIAA Aerospace Sciences Meeting and Exhibit, 2002, pp. 1-9.

${ }^{36}$ Suzuki, T., Bodony, D., Ryu, J., and Lele, S. K., "Noise Sources of High-Mach-number Jets at Low Frequencies Studied with a Phased-array Approach Based on LES Database," Annual research briefs, Center for Turbulence Research, NASA Ames and Stanford University, 2007, http://www.stanford.edu/group/ctr/ResBriefs/ARB07.html.

${ }^{37}$ Sirovich, L., "Turbulence and the Dynamics of Coherent Structures," Quarterly of Applied Mathematics, Vol. XLV(3), 1987, pp. 561-590. 817 .

${ }^{38}$ Chatterjee, A., "An introduction to the proper orthogonal decomposition," Current science, Vol. 78, No. 7, 2000, pp. 808-

${ }^{39}$ Rowley, C. W., Modeling, simulation, and control of cavity flow oscillations, Ph.D. thesis, California Institute of Technology, July 2001.

${ }^{40}$ Hileman, J. I., Thurow, B. S., Caraballo, E. J., and Samimy, M., "Large-scale structure evolution and sound emission in high-speed jets: real-time visualization with simultaneous acoustic measurements," Journal of Fluid Mechanics, Vol. 544, 2005, pp. 277-307.

${ }^{41}$ Hileman, J. and Samimy, M., "Turbulence structures and the acoustic far field of a Mach 1.3 jet," AIAA journal, Vol. 39, No. 9, 2001, pp. 1716-1727.

${ }^{42}$ Goldstein, M., "The low frequency sound from multipole sources in axisymmetric shear flows, with applications to jet noise," Journal of Fluid Mechanics, Vol. 70, No. 03, 1975, pp. 595-604.

${ }^{43}$ Goldstein, M., "High frequency sound emission from moving point multipole sources embedded in arbitrary transversely sheared mean flows," Journal of Sound and Vibration, Vol. 80, No. 4, 1982, pp. 499-522.

${ }^{44}$ Proudman, I., "The generation of noise by isotropic turbulence," Proceedings of the Royal Society of London. Series A. Mathematical and Physical Sciences, Vol. 214, No. 1116, 1952, pp. 119-132.

${ }^{45}$ Panda, J., "Identification of Noise Sources in High Speed Jets via Correlation Measurements - A Review," 11th (AIAA/CEAS) Aeroacoustics Conference, May 2005, AIAA 2005-2844.

${ }^{46}$ Ochmann, M., "The Source Simulation Technique for Acoustic Radiation Problems," Acustica, Vol. 81, 1995 , pp. 512-527. 